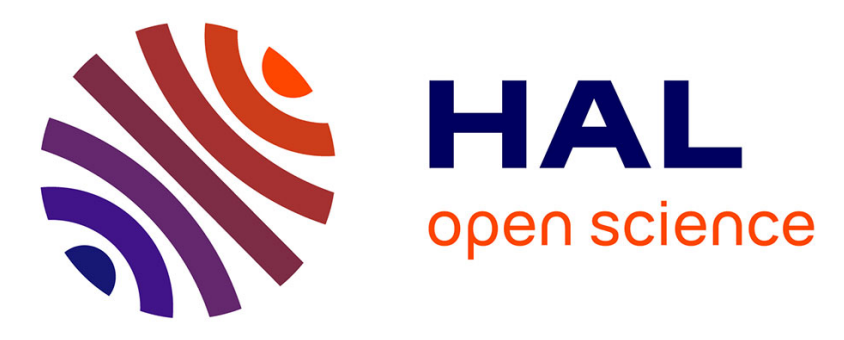

\title{
Synthesis, Structure and Hirshfeld Surface Analysis of a New Iron Complex [Fe(N4Py)(tcnspr)] (tcnspr)
}

Zouaoui Setifi, Hela Ferjani, Christian Jelsch, Christopher Glidewell, Necmi Dege, Fatima Setifi

\section{- To cite this version:}

Zouaoui Setifi, Hela Ferjani, Christian Jelsch, Christopher Glidewell, Necmi Dege, et al.. Synthesis, Structure and Hirshfeld Surface Analysis of a New Iron Complex [Fe(N4Py)(tcnspr)] (tcnspr). Journal of Inorganic and Organometallic Polymers and Materials, 2021, 31, pp.3054-3061. 10.1007/s10904021-01898-2 . hal-03131756

\section{HAL Id: hal-03131756 https://hal.science/hal-03131756}

Submitted on 4 Feb 2021

HAL is a multi-disciplinary open access archive for the deposit and dissemination of scientific research documents, whether they are published or not. The documents may come from teaching and research institutions in France or abroad, or from public or private research centers.
L'archive ouverte pluridisciplinaire HAL, est destinée au dépôt et à la diffusion de documents scientifiques de niveau recherche, publiés ou non, émanant des établissements d'enseignement et de recherche français ou étrangers, des laboratoires publics ou privés. 
Published in Journal of Inorganic and Organometallic Polymers and Materials (2021) p 1-8.

https://doi.org/10.1007/s10904-021-01898-2

\title{
Synthesis, Structure and Hirshfeld Surface Analysis of a New Iron Complex [Fe(N4Py)(tenspr)] (tenspr)
}

\author{
Zouaoui Setifia ${ }^{\mathrm{a}, \mathrm{b}}$, Hela Ferjani ${ }^{\mathrm{c}, *}$, Christian Jelsch $^{\mathrm{d}}$, Christopher Glidewell ${ }^{\mathrm{e}}$, Necmi Dege ${ }^{\mathrm{f}}$, \\ Fatima Setifi ${ }^{\mathrm{a}, *}$
}

${ }^{a}$ Laboratoire de Chimie, Ingénierie Moléculaire et Nanostructures (LCIMN), Université Ferhat Abbas Sétif 1, Sétif 19000, Algeria

${ }^{b}$ Département de Technologie, Faculté de Technologie, Université 20 Août 1955-Skikda, Skikda 21000, Algeria

${ }^{c}$ Chemistry Department, College of Science, IMSIU (Imam Mohammad Ibn Saud Islamic University), Riyadh 11623, Kingdom of Saudi Arabia

${ }^{d} C_{R M}{ }^{2}, C N R S$, Institut Jean Barriol, Université de Lorraine, 54000, Nancy, France

${ }^{e}$ School of Chemistry, University of St Andrews, St Andrews, Fife, KY16 9ST, UK

${ }^{f}$ Ondokuz Mayls University, Faculty of Arts and Sciences, Department of Physics, 55139, Samsun, Turkey

*Corresponding authors E-mail addresses: hhferjani@,imamu.edu.sa(H. Ferjani),

fat_setifi@yahoo.fr(F. Setifi)

\begin{abstract}
We report the synthesis, crystal structure and Hirshfeld surface analysis of Fe(II) complex based on a pentadentate N4Py (N4Py = N,N-bis(2-pyridylmethyl)-N-(bis-2-pyridylmethyl)amine) and polynitrile tenspr (1,1,3,3-tetracyano-2-thioethylpropenide) ligands, [Fe(N4Py)(tcnspr)](tcnspr) (I). The X-ray result, indicates that (I) is low-spin Fe(II) complex. The tcnspr molecule in the complex exhibits disorder over two sets of atomic sites having occupancies of 0.681 (6) and 0.319 (6). The crystal packing of (I) is established by weak intermolecular C-H...N/S hydrogen bond, C-N... $\pi$ and stacking interactions. Hirshfeld surface analysis employing three-dimensional molecular surface contours and enrichment ratio calculations have been used to understand the intermolecular interactions present in the structure.
\end{abstract}




\section{Keywords}

Crystal structure, Polynitrile anion, Disorder, Hydrogen bonding, $\pi$-stacking interactions, Supramolecular assembly, Hirshfeld surface analysis.

\section{Introduction}

As a consequence of their ability to link metal ions in a variety of different ways, organic polynitrile anions, either functioning alone or in combination with neutral co-ligands, provide opportunities for the generation of molecular architectures with varying dimensions and topologies [1-7]. The presence of other potential donor groups such as those derived from $-\mathrm{OH},-\mathrm{SH}$ or $\mathrm{NH}_{2}$, together with their rigidity and electronic delocalization, mean that polynitrile anions can also lead to new magnetic and luminescent coordination polymers based on transition-metal ions [8-12]. Furthermore, the use of polynitrile anions for the synthesis of interesting discrete and polymeric bistable materials has been described [13-16]. It was during the course of attempts to prepare such complexes with N,N-bis(2-pyridylmethyl)-N-bis(2-pyridyl)methylamine (N4Py) as a co-ligand that the title complex, (I), was unexpectedly obtained. Herein, the crystal and molecular structure of $\mathbf{I}$ are described. A study complemented by an analysis of the molecular packing by calculating the Hirshfeld surfaces study.

\section{EXPERIMENTAL}

\section{Synthesis}

All chemical reagents and solvents are commercially available and were used without further purification. Compound (I) was prepared using a mixture of iron(II) sulfate heptahydrate (28 mg, $0.1 \mathrm{mmol})$, N4Py $(37 \mathrm{mg}, 0.1 \mathrm{mmol})$ and Ktcnspr $(48 \mathrm{mg}, 0.2 \mathrm{mmol})$ in mixed solvents of water/methanol $(3: 1 \mathrm{v} / \mathrm{v}, 20 \mathrm{ml})$. The mixture was sealed in a Teflon-lined autoclave and held at $403 \mathrm{~K}$ for $3 \mathrm{~d}$, and then cooled to room temperature at a rate of $10 \mathrm{~K}$ per hour (yield $27 \%$ ). Red prismatic crystals of (I) suitable for single-crystal X-ray diffraction analysis were selected. 


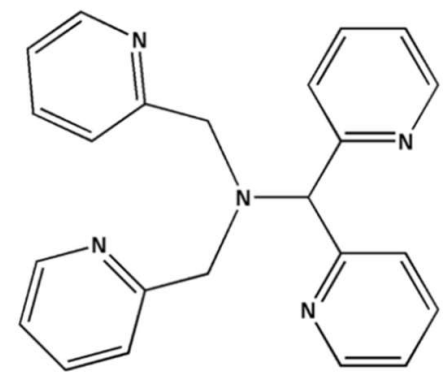

(N4Py)

(a)

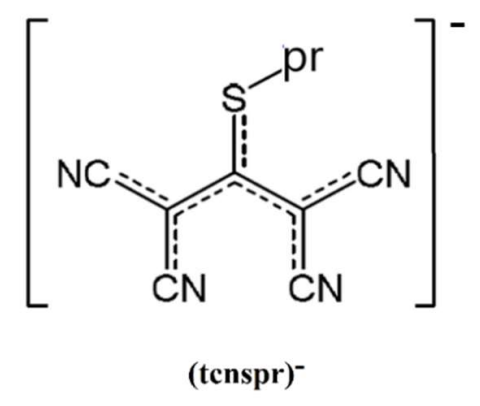

(b)

Figure 1. Schemes of (N4Py) (a) and (tcnspr) (b) molecules.

\section{X-Ray Diffraction Analysis}

Molecular and crystal structure of compound (I) was determined by single crystal X-ray diffraction technique. X-ray diffraction data collection was carried out on a STOE IPDS 2 [17] diffractometer with graphite monochromatized Mo $\mathrm{K} \alpha$ radiation $(\lambda=0.71073 \AA)$. Semiempirical absorption correction was performed with SADABS [18]. Crystal structure was solved by direct method (SHELXS) [19] and refined by full matrix least squares techniques (SHELXL-2014/7) [20]. All non-hydrogen atoms were refined anisotropically. $\mathrm{H}$ atoms were located in difference-Fourier maps but introduced in calculated positions and treated as riding on their parent atoms, with $\mathrm{C}-\mathrm{H}$ $=0.93$ and $0.97 \AA$ with $\mathrm{U}_{\text {iso }}(\mathrm{H})=1.2 \mathrm{U}_{\mathrm{eq}}(\mathrm{C})$. The disordered (tcnspr) molecule was refined by splitting atoms over two positions (A and B parts) with occupancy factors of 0.681 (6) and 0.319 (6). Constraints EXYZ and EADP on bond lengths were applied. The structural diagrams were drawn with Diamond 3 [21]. Data collection and refinement parameters for the complex (I) are summarized in Table 1. CCDC 2042161 contains the supplementary crystallographic data for this paper. These data can be obtained free of charge via www.ccdc.cam.ac.uk/data_request/cif, or by emailing data_request@ccdc.cam.ac.uk, or by contacting The Cambridge Crystallographic Data Centre, 12, Union Road, Cambridge CB2 1EZ, UK; fax: +44 1223336033. 


\section{RESULTS AND DISCUSSION}

\section{Crystal structure description}

Complex (I) crystallizes in triclinic crystal system having space group $P-1$. The molecular structure of compound (I) is shown in Fig. 2. It contains one cation $[\mathrm{Fe}(\mathrm{N} 4 \mathrm{Py})(\mathrm{tcnspr})]^{+}$and one non-coordinated anion (tcnspr)- The (tcnspr) molecule in the complex exhibits disorder over two sets of atomic sites with occupancies of 0.681 (6) and 0.319 (6), but the molecular conformations of the two disorder moieties are very similar and we comment in detail only on that of the major one (Solid bonds). The iron (II) center is six-coordinated by four $\mathrm{N}_{\mathrm{pyr}}$ atoms and one tertiary nitrogen donor of N4Py ligand, occupying the apical position. The sixth coordination site is occupied by (tcnspr) coordinated molecule. The four pyridine rings and (tcnspr) molecule are all positioned perpendicular to the basal plane of coordination (Fig. 2). The Fe-N ligand distances range from 1.957 (2) $\AA$ for Fe-N2 to 1.968 (2) $\AA$ for Fe-N4. The Fe-N6 bond of the polynitrile anion is the shorter than nitrogen atoms of N4Py ligand (Table 2). Overall, the Fe-N bond lengths are comparable to those found for other low-spin iron(II) complexes [22-24]. The band distances and angles within two different ligands are in good agreement with those observed in similar compounds $[\mathbf{2 5 , 2 6 ]}$.

The careful analysis of the shortest inter-molecular contacts (hydrogen bonding and $\pi$-stacking contacts) in (I) reveals that the moieties are linked together by weak $\mathrm{C}-\mathrm{H} \cdots \mathrm{N}$ hydrogen bonds (Table 3) involving the carbons of tertiary amine and pyridine groups and the terminal nitrogen atoms from the adjacent (tcnspr) polynitrile anions, (Figure 3b and d). Another week hydrogen bond $\mathrm{C}-\mathrm{H} \ldots \mathrm{S}$ is also observed between $\mathrm{C} 2$ of amine groups and sulfur atom of adjacent coordinated (tcnspr) molecule within the cation (Table 3).

Furthermore, a remarkable supramolecular assembly is formed by means of a combination of common and uncomment $\pi-\pi$ interactions, which is described by [27-29], where the donor $\pi$ system is a triple bond within the polynitrile anion $\pi(\mathrm{C} \equiv \mathrm{N})$. Thus, the polynitrile anions (tcnspr) chains are connected to each other through hydrogen bonds C-H..N (3.538 (2) $\AA$ ) and by van der Waals contacts C-H... $\pi$ (3.119 (2) -3.957 (3) $\AA$ ), $\pi \ldots \pi$ (centroid-centroid distances $=4.210$ (3) $\AA ̊$ ) and N... $\pi$ interactions (3.389 (2) $\AA$ ), see Figure 3c. The $\left[\mathrm{Fe}\left(\mathrm{N}_{4} \mathrm{Py}\right)(\mathrm{tcnspr})\right]^{+}$cations are interconnect to each other by weak $\mathrm{C}-\mathrm{H}$... $\mathrm{N}$ hydrogen bonding and by $\pi \ldots . \pi$ interactions between coordinated (tcnspr) molecule (centroid-centroid distances $\sim 4.3$ (3) $\AA$ ) (Figure 2.d). 
There is also a single C-N... $\left(\right.$ pyridine) interactions, with dimensions N12A...Cg $4^{\mathrm{i}} \sim 3.1358(3) \AA$ and $\mathrm{N} 12 \mathrm{~B} \ldots \mathrm{Cg} 4^{\mathrm{i}} \sim 3.4836(3) \AA$, where $\mathrm{Cg} 4$ represents the centroid of the pyridine ring $(\mathrm{N}(5)$, $\mathrm{C}(19), \mathrm{C}(20), \mathrm{C}(21), \mathrm{C}(22), \mathrm{C}(23))$ and the symmetry code $\mathrm{i}=(\mathrm{x}, \mathrm{y}, \mathrm{z})$ (Figure 3b). Such various intermolecular contacts lead to a supramolecular alternated chains oriented along the $b$ axis (Fig. 3a).

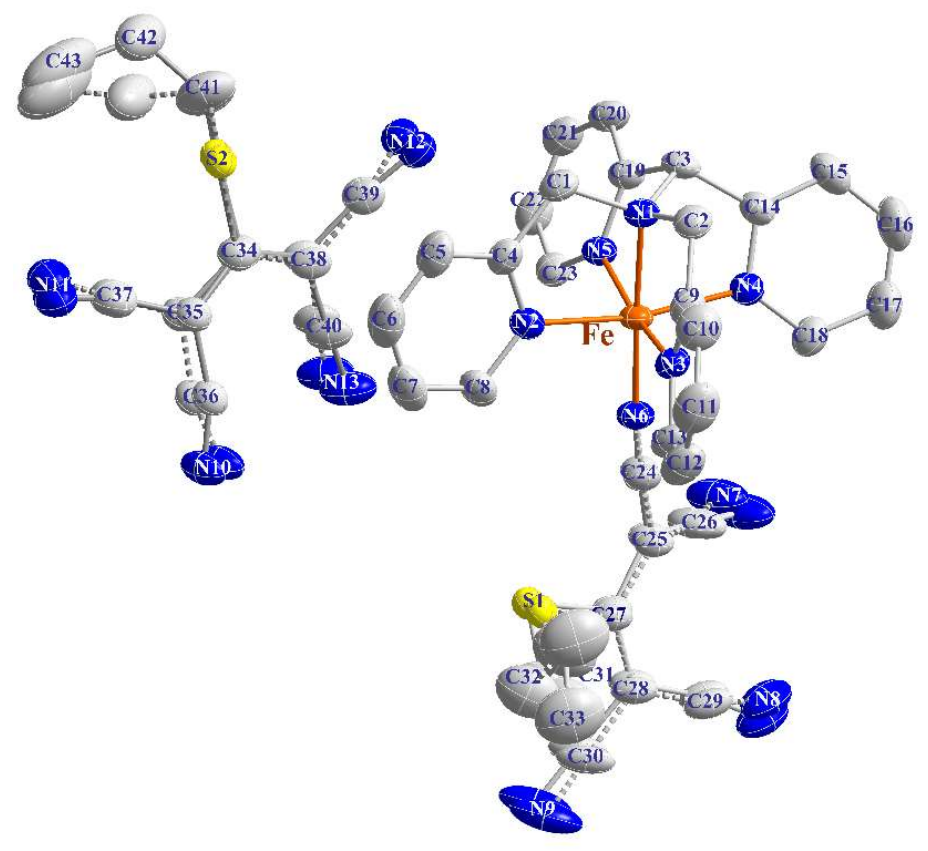

Figure 2. The molecular structure of (I), showing the atom-labelling scheme and the two disorder components, whose occupancies are 0.681(6) (solid bonds) and 0.319 (6) (broken bonds). Displacement ellipsoids are drawn at the $30 \%$ probability level. Hydrogen atoms omitted for clarity. 


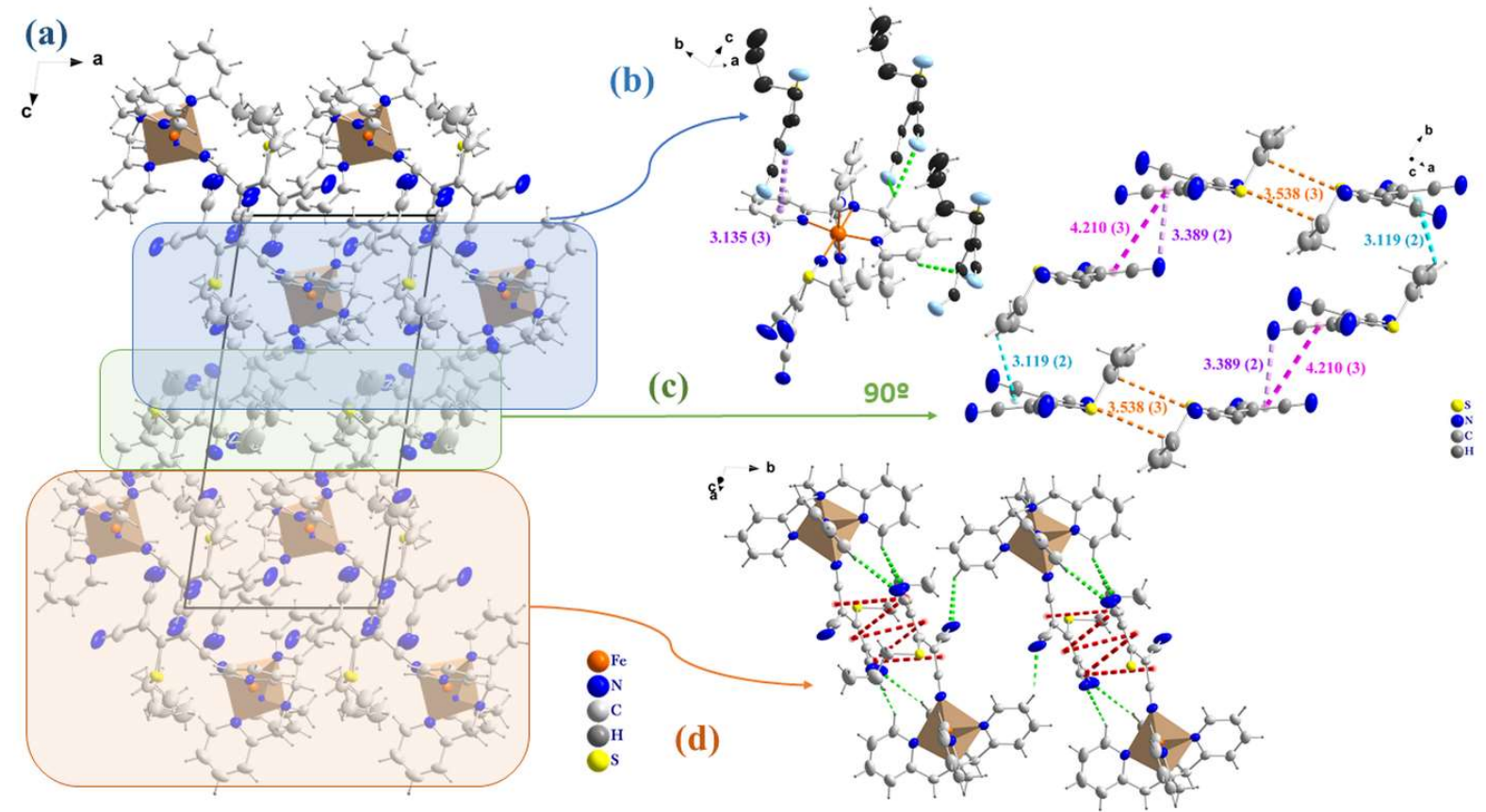

Figure 3: (a) View along $b$ axis of the packing diagram, (b) Hydrogen bonding (green dashed line) and $\mathrm{N} . . . \pi$ (clear violet dashed line) interactions between $\left[\mathrm{Fe}\left(\mathrm{N}_{4} \mathrm{Py}\right)(\mathrm{tcnspr})\right]^{+}$cations and (tcnspr) ${ }^{-}$ anions, (c) A scheme of (tenspr) ${ }^{-}$anions formed by $\mathrm{C}-\mathrm{H}^{\cdots} \mathrm{N}$ hydrogen bonds (orange dashed line), $\mathrm{C}-\mathrm{H} \cdots \pi$ (Cyan dashed line), $\mathrm{N} \cdots \pi$ (clear violet dashed line) and $\pi \cdots \pi$ (pink dashed line) interactions, (d) A scheme of $\left[\mathrm{Fe}\left(\mathrm{N}_{4} \mathrm{Py}\right)(\text { tenspr) }]^{+}\right.$cations chains formed by $\mathrm{C}-\mathrm{H} \cdots \mathrm{N}$ (green dashed line) and $\pi(\mathrm{C} \equiv \mathrm{N}) \ldots \pi(\mathrm{C} \equiv \mathrm{N})$ (red dashed line) interactions. 
Table 1. Selected Single-Crystal Data and Structure Refinement Parameters for (I)

\begin{tabular}{|c|c|}
\hline Empirical formula & $\mathrm{C}_{43} \mathrm{H}_{35} \mathrm{FeN}_{13} \mathrm{~S}_{2}$ \\
\hline Formula weight $(\mathrm{g} / \mathrm{mol})$ & 853.81 \\
\hline Crystal system, space group & Triclinic, $\mathrm{P}-1$ \\
\hline $\mathrm{a}(\AA)$ & $9.7573(7)$ \\
\hline $\mathrm{b}(\AA)$ & $11.3795(9)$ \\
\hline$c(\AA)$ & $20.1483(17)$ \\
\hline$\alpha\left(^{\circ}\right)$ & $100.527(7)$ \\
\hline$\beta\left(^{\circ}\right)$ & $96.823(6)$ \\
\hline$\gamma\left({ }^{\circ}\right)$ & $97.529(6)$ \\
\hline $\mathrm{V}\left(\AA^{3}\right)$ & $2157.0(3)$ \\
\hline$Z$ & 2 \\
\hline$\mu\left(\mathrm{mm}^{-1}\right)$ & 0.49 \\
\hline$D_{\mathrm{x}}\left(\mathrm{Mg} \mathrm{m}^{-3}\right)$ & 1.315 \\
\hline$F(000)$ & 884 \\
\hline Crystal size (mm) & $0.62 \times 0.33 \times 0.17$ \\
\hline Crystal habit & Red Rod \\
\hline$\theta_{\min } / \theta_{\max }(\mathrm{deg})$ & $1.8 / 26.6$ \\
\hline Measured reflections & 21644 \\
\hline Independent reflections & 8910 \\
\hline Observed refl. with $\mathrm{I}>2 \sigma(\mathrm{I})$ & 5964 \\
\hline $\mathrm{R}_{\text {int }}$ & 0.033 \\
\hline Data/restraints/parameters & $8910 / 62 / 625$ \\
\hline $\mathrm{R}\left[\mathrm{F}^{2}>2 \sigma\left(\mathrm{F}^{2}\right)\right]$ & 0.048 \\
\hline $\mathrm{wR}\left(\mathrm{F}^{2}\right)$ & 0.126 \\
\hline $\mathrm{GooF}=\mathrm{S}$ & 0.96 \\
\hline$\Delta \rho_{\max } / \Delta \rho_{\min }\left(\mathrm{e} . \AA^{-3}\right)$ & $0.60 /-0.29$ \\
\hline
\end{tabular}


Table 2: Selected bond lengths $(\AA)$ and angles $\left({ }^{\circ}\right)$ for (I)

\begin{tabular}{|l|l|}
\hline Fe-N6B & $1.907(2)$ \\
\hline Fe-N6A & $1.907(2)$ \\
\hline Fe-N3 & $1.957(2)$ \\
\hline Fe-N5 & $1.958(2)$ \\
\hline Fe-N1 & $1.962(2)$ \\
\hline Fe-N2 & $1.967(2)$ \\
\hline Fe-N4 & $1.968(2)$ \\
\hline N6B-Fe-N3 & $97.32(10)$ \\
\hline N6A-Fe-N3 & $97.32(10)$ \\
\hline N6B-Fe-N5 & $94.24(9)$ \\
\hline N6A-Fe-N5 & $94.24(9)$ \\
\hline N3-Fe-N5 & $168.43(9)$ \\
\hline N6B-Fe-N1 & $175.79(9)$ \\
\hline N6A-Fe-N1 & $175.79(9)$ \\
\hline N3-Fe-N1 & $85.32(9)$ \\
\hline N5-Fe-N1 & $83.15(8)$ \\
\hline N6B-Fe-N2 & $98.64(10)$ \\
\hline N6A-Fe-N2 & $98.64(10)$ \\
\hline N3-Fe-N2 & $86.13(9)$ \\
\hline N5-Fe-N2 & $91.99(9)$ \\
\hline N1-Fe-N2 & $84.78(9)$ \\
\hline N6B-Fe-N4 & $93.93(9)$ \\
\hline N6A-Fe-N4 & $93.93(9)$ \\
\hline N3-Fe-N4 & $92.14(9)$ \\
\hline N5-Fe-N4 & $87.21(9)$ \\
\hline N1-Fe-N4 & $82.66(9)$ \\
\hline N2-Fe-N4 & $167.42(9)$ \\
\hline & \\
\hline
\end{tabular}


Table 3: Hydrogen-bond geometry in (I)

\begin{tabular}{|c|c|c|c|c|}
\hline D-H $\cdots A$ & D-H & $\mathbf{H} \cdots \mathbf{A} / \AA$ & $\mathbf{D} \cdots \mathbf{A} / \AA$ & $\mathbf{D}-\mathbf{H} \cdots \mathbf{A} /^{\circ}$ \\
\hline C1-H1A $\cdots$ N12A & 0.97 & 2.59 & $3.37(3)$ & 137 \\
\hline C1-H1B $\cdots N^{10} A^{i}$ & 0.97 & 2.70 & $3.64(3)$ & 163 \\
\hline C1-H1B $\cdots N^{N 10 B}{ }^{i}$ & 0.97 & 2.59 & $3.52(4)$ & 162 \\
\hline C7-H7 $\cdots N^{N 11 A^{i i}}$ & 0.93 & 2.50 & $3.30(3)$ & 145 \\
\hline $\mathrm{C} 2-\mathrm{H} 2 \mathrm{~A} \cdots \mathrm{N} 10 \mathrm{~A}^{\mathrm{i}}$ & 0.97 & 2.69 & $3.626(15)$ & 162 \\
\hline $\mathrm{C} 2-\mathrm{H} 2 \mathrm{~A} \cdots \mathrm{N} 10 \mathrm{~B}^{\mathrm{i}}$ & 0.97 & 2.44 & $3.39(2)$ & 168 \\
\hline $\mathrm{C} 2-\mathrm{H} 2 \mathrm{~B} \cdots \mathrm{S} 1 \mathrm{~A}^{\mathrm{i}}$ & 0.97 & 3.02 & $3.745(5)$ & 133 \\
\hline C10-H10 $\cdots N^{11} A^{i i i}$ & 0.93 & 2.53 & $3.43(6)$ & 162 \\
\hline C10-H10 $\cdots$ N11B & 0.93 & 2.50 & $3.37(9)$ & 155 \\
\hline C13-H13 $\cdots$ N6A & 0.93 & 2.69 & $3.156(4)$ & 112 \\
\hline C3-H3 $\cdots N^{N} 13 B^{i}$ & 0.98 & 2.54 & 3.367 (16) & 143 \\
\hline C15-H15 $\cdots$ N7A $^{i v}$ & 0.93 & 2.69 & $3.561(12)$ & 157 \\
\hline C15-H15 $\cdots$ N7B $^{\text {iv }}$ & 0.93 & 2.64 & $3.47(3)$ & 150 \\
\hline $\mathrm{C}^{22-H 22} \cdots \mathrm{N}^{2} \mathrm{~A}^{\mathrm{v}}$ & 0.93 & 2.65 & $3.404(18)$ & 138 \\
\hline
\end{tabular}

Symmetry codes: (i) $\mathrm{x}+1, \mathrm{y}, \mathrm{z}$; (ii) $-\mathrm{x},-\mathrm{y}+1,-\mathrm{z}+1$; (iii) $-\mathrm{x}+1,-\mathrm{y}+1,-\mathrm{z}+1$; (iv) $-\mathrm{x}+1,-\mathrm{y}+1,-\mathrm{z}$; (v) -x, -y+1, -z.

\section{Hirshfeld Surface analysis}

Analysis of Hirshfeld surface represents a major analysis tool enabling supramolecular chemists and crystal engineers to gain insight into understanding the crystal packing. The Hirshfeld surfaces are constructed based on the atomic electron densities.

The MoProViewer software [30] was used to quantify and visualize all intermolecular contacts in the main conformation of the molecular structure and compute the enrichment of contact types. The enrichment ratio $E_{\mathrm{XY}}$ for a pair of chemical elements $(\mathrm{X}, \mathrm{Y})$ is defined as the ratio between the percentage of actual contacts in the crystal $\left(C_{\mathrm{XY}}\right)$ and that of equi-distributed contacts $R_{\mathrm{XY}}$. An enrichment ratio larger or smaller than unity reveals that the contact type is over or underrepresented, respectively [31].

The Hirshfeld surface was computed around the $\left[\mathrm{Fe}\left(\mathrm{N}_{4} \mathrm{Py}\right)(\mathrm{tcnspr})\right]^{+}$complex cation and (tcnspr) ${ }^{-}$ anion (Table 4). Hydrogen is the most abundant species on the surface, followed by carbon and nitrogen.

The surface is constituted a weak hydrogen bond donor $(\mathrm{H}-\mathrm{C})$ and three hydrogen bond acceptors of different strengths $(\mathrm{C}<\mathrm{S}<\mathrm{N})$. The strongest $\mathrm{N}$...H contacts are indeed the most enriched $\left(E_{\mathrm{NH}}=1.7\right)$ followed by S...H $\left(E_{\mathrm{SH}}=1.7\right)$ while the weakest H...C $(\mathrm{H} \ldots \pi)$ are slightly disfavored in the crystal packing. The N...H and C...H hydrogen bonds are the most represented contacts in the crystal packing. Hydrophobic H...H and C...C self-contacts are the next well represented due to the abundance of these elements on the surface. Besides the H...N and H...S hydrogen bonds, 
C...C represents the only significantly enriched contact at $E_{\mathrm{CC}}=1.36$, due to interactions between the $\mathrm{C}-\mathrm{C} \equiv \mathrm{N}$ groups with the $\mathrm{C}_{5} \mathrm{~N}$ aromatic rings.
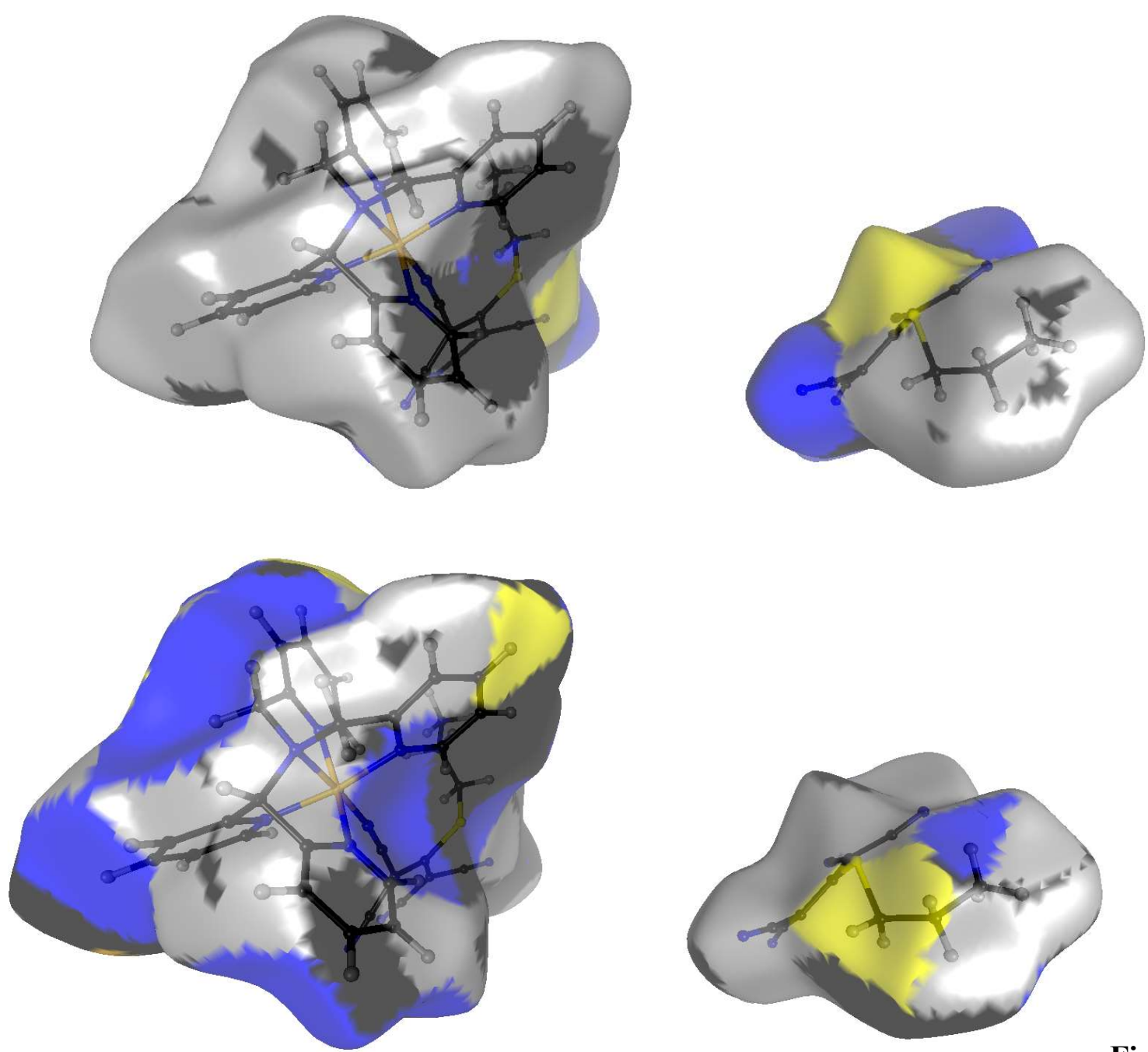

Figure

4. Hirshfeld surface around the $\left[\mathrm{Fe}\left(\mathrm{N}_{4} \mathrm{Py}\right)(\mathrm{tcnspr})\right]^{+}$complex cation and (tcnspr) ${ }^{-}$anion. The surface is coloured according to the interior (a) and exterior (b) atom contributing most to the electron density. Carbon: dark grey; Hydrogen: grey; Sulphur: yellow; Nitrogen: blue. 
Table 4: Hirshfeld contact surfaces and enrichment ratios for (I)

\begin{tabular}{|lcccc|}
\hline atom & $\mathrm{C}$ & $\mathrm{N}$ & $\mathrm{S}$ & $\mathrm{H}$ \\
surface\% & 29.6 & 19.7 & 4.3 & 46.2 \\
\hline C & $\mathbf{1 2 . 0}$ & & & \\
N & 8.2 & 0.4 & & \% contacts \\
S & 2.9 & 0.2 & 0.0 & \\
H & $\mathbf{2 4 . 0}$ & $\mathbf{3 1 . 1}$ & 5.8 & $\mathbf{1 5 . 1}$ \\
\hline C & $\mathbf{1 . 3 6}$ & & & \\
N & 0.68 & 0.10 & & enrichment \\
S & 1.11 & 0.09 & 0.13 & \\
H & 0.89 & $\mathbf{1 . 7 0}$ & $\mathbf{1 . 4 2}$ & 0.73 \\
\hline
\end{tabular}

Table 4. Chemical proportions on the Hirshfeld surface around the iron complex and the organic moiety in the crystal packing of the title compound. The actual contact $C x y$ by chemical types and their enrichment $E_{\mathrm{xy}}$ are then given. The major contacts and the most enriched are highlighted in bold.

\section{References}

1. C. Atmani, F. Setifi, S. Benmansour, S. Triki, M. Marchivie, J.-Y. Salaün, \& C. J. GómezGarcía., Inorganic Chemistry Communications, 11(8), 921-924 (2008).

2. S. Benmansour, F. Setifi, S. Triki, J.-Y. Salaün, F. Vandevelde, J. Sala-Pala, C. J. GómezGarcía, T. Roisnel., European Journal of Inorganic Chemistry, 2007(1), 186-194 (2007).

3. S. Benmansour, F. Setifi, C. J. Gómez-García, S. Triki, E. Coronado \& J.-Y. Salaün., Journal of Molecular Structure, 890(1-3), 255-262 (2008).

4. S. Benmansour, F. Setifi, S. Triki, \& C. J. Gómez-García., Inorganic Chemistry, 51(4), 23592365(2012)

5. Z. Setifi, F. Lehchili, F. Setifi, A. Beghidja, S. W. Ng \& C. Glidewell., Acta Crystallogr., Sect.

C: Struct. Sci. 70(3). 338-341 (2014).

6. F. Lehchili, F. Setifi, X. Liu, A. Saneei, M.Kučeráková, Z. Setifi, M. Dušek, M. Poupon, M. Pourayoubi, J. Reedijk., Polyhedron, 131, 27-33 (2017).

7. S. Benmansour, F. Setifi, C. J. Gómez-García, S. Triki, E. Coronado., Inorganica Chimica Acta, 361(14-15), 3856-3862 (2008).

8. F. Setifi, S. Benmansour, M. Marchivie, G. Dupouy, S. Triki, J. Sala-Pala, J-Y. Salaün, C. J. Gómez-García, S. Pillet, C. Lecomte, E. Ruiz., Inorganic Chemistry, 48(4), 1269-1271 (2009).

9. C. Yuste, A. Bentama, N. Marino, D. Armentano, F. Setifi, S. Triki, F. Lloret, M. Julve., Polyhedron, 28(7), 1287-1294 (2009). 
10. F. Setifi, S. Benmansour, S. Triki, C. J. Gómez-García, M. Marchivie, J.-Y. Salaün, \& M. Mustapha., Inorganica Chimica Acta, 360(13), 3879-3886 (2007).

11. A. Addala, M. Poupon, S. Bernès, G. S. Kürkçüoğlu, X. Liu, F. Lehchili, M. Kučeráková, M. Dušek, F. Setifi, Z.Setifi, J. Reedijk, Polyhedron. 170, 271-277 (2019).

12. Y. S. Kayukov, S. V. Karpov, A. A. Grigor'ev, O. E. Nasakin, V. A. Tafeenko, K. A. Lyssenko, A. V. Shapovalov, E. A. Varaksina., Dalton Transactions, 46(48), 16925-16938 (2017).

13. S. Benmansour, C. Atmani, F. Setifi, S.Triki, M. Marchivie, \& C. J. Gómez-García, Coordination Chemistry Reviews, 254(13-14), 1468-1478 (2010).

14. F. Setifi, E. Milin, C. Charles, F. Thétiot, S.Triki, \& C. J. Gómez-García., Inorganic Chemistry, 53(1), 97-104 (2013).

15. F. Setifi, C. Charles, S. Houille, F. Thétiot, S. Triki, C. J. Gómez-García, \& S. Pillet., Polyhedron, 61, 242-247 (2013).

16. B. Benaicha, K. Van Do, A. Yangui, N. Pittala, A. Lusson, M. Sy, G. Bouchez, H. Fourati, C. J. Gómez-García, S. Triki, K. Boukheddaden., Chem. Sci., 2019, 10, 6791-6798 (2019).

17. Stoe \& Cie (2002). X-AREA and X-RED32. Stoe \& Cie GmbH, Darmstadt, Germany.

18. Sheldrick, G. M. (2003). SADABS. Bruker AXS Inc., Madison, Wisconsin, USA.

19. Sheldrick, G. M. (2008). Acta Cryst. A64, 112-122.

20. Sheldrick, G. M. (2015). Acta Cryst. C71, 3-8.

21. Brandenburg, K. (2006). DIAMOND. Crystal Impact GbR, Bonn, Germany.

22. A. Draksharapu, Q. Li, H. Logtenberg, T. A. Van den Berg, A. Meetsma, J. S. Killeen, W. R. Browne., Inorg. Chem, 51(2), 900-913 (2011).

23. G. Gerard Roelfes, M. Lubben, K. Chen, R Y. N. Ho, A. Meetsma, S. Genseberger, R. M. Hermant, R. Hage, S. K. Mandal, V. G. Jr. Young, Y. Zang, H. Kooijman, A. L. Spek, L. Jr. Que, B. L. Feringa., Inorg. Chem, 38, 1929-1936 (1999).

24. G. M. E. Posse, M. A. Juri, P. J. Aymonino, L. E. Piro,; H. A. Negri, E. E. Castellano., Inorg. Chem, 23, 948-952 (1984).

25. M. Lubben, A. Meetsma, E. C. Wilkinson, B. Feringa, \& L. Que., Angewandte Chemie International Edition in English, 34(13-14), 1512-1514 (1995).

26. L. R. Widger, Y. Jiang, A. C. McQuilken, T. Yang, M. A. Siegler, H. Matsumura, D. P. Goldberg., Dalton Transactions, 43(20), 7522 (2014). 
27. L. Bofill, R. Prohens, R. Barbas, \& A. Frontera, (2020). Crystal Growth \& Design. 20(10), 6691-6698 (2020).

28. M. Savastano, C. Bazzicalupi, P. Mariani, A. Bianchi., Molecules, 23(3), 572 (2018).

29. Z. Setifi, N. Cubillán, C. Glidewell, S. K. Nayak, M. Morales-Toyo, R. Khajavian, M. Mirzaei., Journal of Molecular Structure, 129438 (2020).

30. B. Guillot, E. Enrique, L. Huder, C. Jelsch, Acta Crystallogr., Sect. A: Struct. Sci. 70. C279 (2014).

31. C. Jelsch, K. Ejsmont, L. Huder., IUCrJ, 1, 119-128 (2014). 


\section{Supplement: CIF FILE}

data_shelx

_audit_creation_method

'SHELXL-2014/7'

_chemical_formula_moiety

'C33 H28 Fe N9 S, C10 H7 N4 S'

_chemical_formula_sum

'C43 H35 Fe N13 S2'

_chemical_formula_weight

853.81

loop_

_atom_type_symbol

_atom_type_description

_atom_type_scat_dispersion_real

_atom_type_scat_dispersion_imag

_atom_type_scat_source

'C' 'C' $0.0033 \quad 0.0016$

'International Tables Vol C Tables 4.2.6.8 and 6.1.1.4'

'H' 'H' $0.0000 \quad 0.0000$

'International Tables Vol C Tables 4.2.6.8 and 6.1.1.4'

'Fe' 'Fe' $0.3463 \quad 0.8444$

'International Tables Vol C Tables 4.2.6.8 and 6.1.1.4'

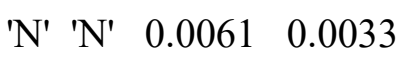

'International Tables Vol C Tables 4.2.6.8 and 6.1.1.4'

'S' 'S' $0.1246 \quad 0.1234$

'International Tables Vol C Tables 4.2.6.8 and 6.1.1.4'

_space_group_crystal_system triclinic

_space_group_IT_number 2 


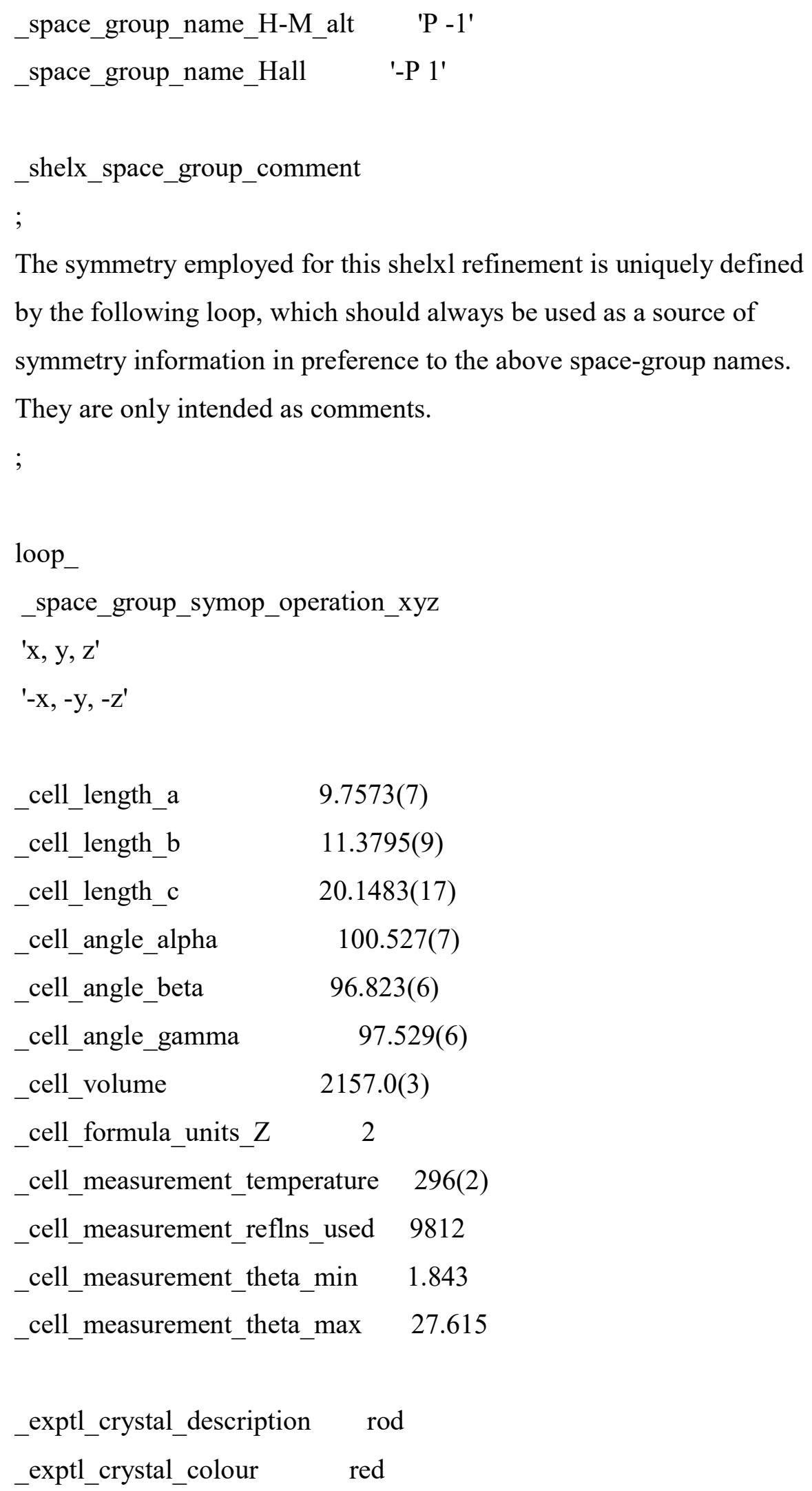


_exptl_crystal_density_meas ?

_exptl_crystal_density_method ?

_exptl_crystal_density_diffrn 1.315

_exptl_crystal_F_000 884

_exptl_transmission_factor_min ?

_exptl_transmission_factor_max ?

_exptl_crystal_size_max 0.620

_exptl_crystal_size_mid 0.333

_exptl_crystal_size_min $\quad 0.170$

_exptl_absorpt_coefficient_mu 0.494

_shelx_estimated_absorpt_T_min 0.749

_shelx_estimated_absorpt_T_max 0.921

_exptl_absorpt_correction_type multi-scan

_exptl_absorpt_correction_T_min 0.796

_exptl_absorpt_correction_T_max 0.919

_exptl_absorpt_process_details 'SADABS (Sheldrick, 2003)'

_diffrn_ambient_temperature 296(2)

_diffrn_radiation_wavelength 0.71073

_diffrn_radiation_type MoKla

_diffrn_source

'fine focus sealed tube'

_diffrn_measurement_device_type

'STOE IPDS 2'

_diffrn_measurement_method_rotation method'

_diffrn_detector_area_resol_mean ?

_diffrn_reflns_number 21644

_diffrn_reflns_av_unetI/netI 0.0438

_diffrn_reflns_av_R_equivalents 0.0332

_diffrn_reflns_limit_h_min -11

_diffrn_reflns_limit_h_max 12

_diffrn_reflns_limit_k_min $\quad-14$ 


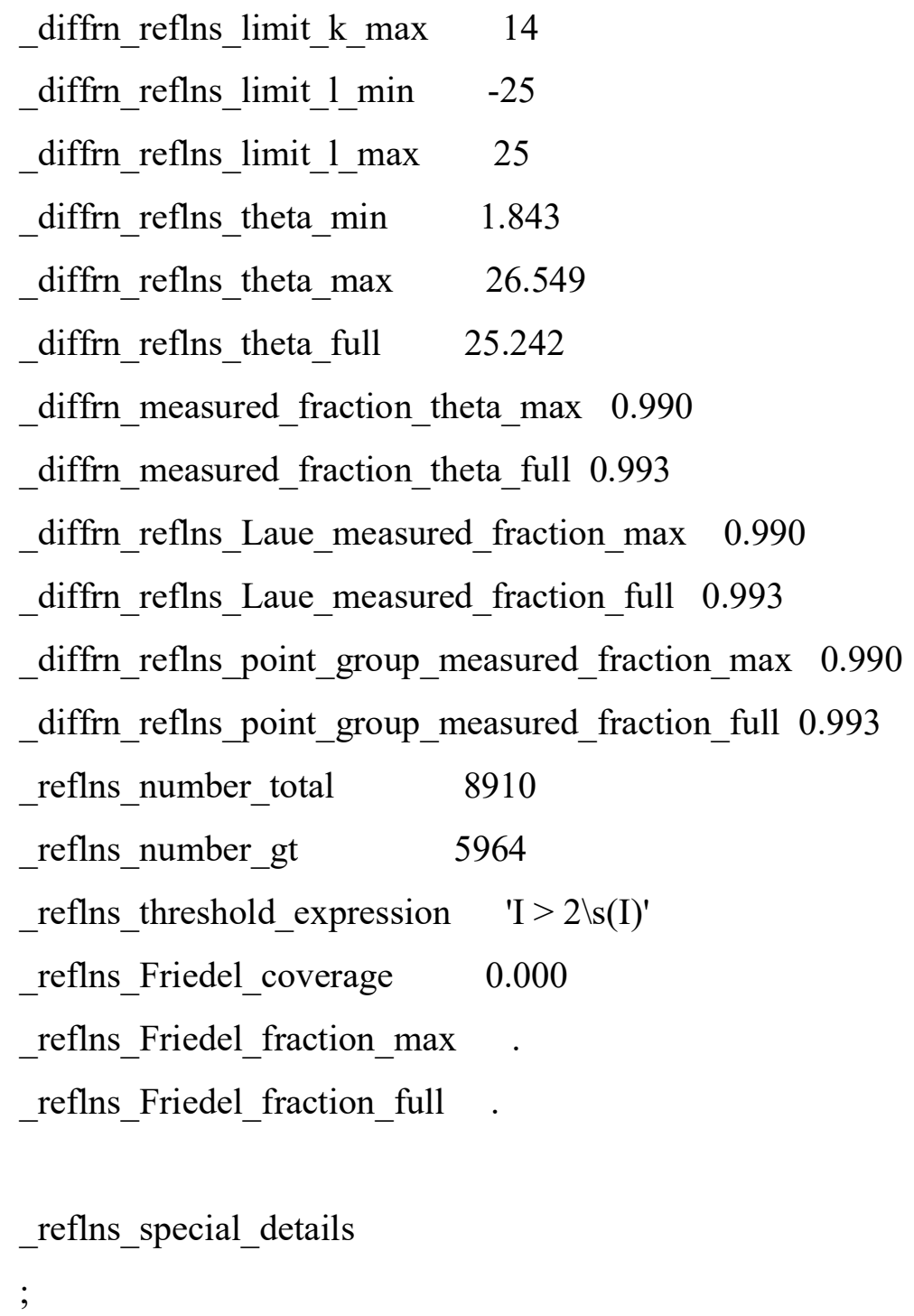

Reflections were merged by SHELXL according to the crystal class for the calculation of statistics and refinement.

_reflns_Friedel_fraction is defined as the number of unique Friedel pairs measured divided by the number that would be possible theoretically, ignoring centric projections and systematic absences.

_computing_data_collection? 
_computing_cell_refinement ?

_computing_data_reduction ?

_computing_structure_solution ?

_computing_structure_refinement 'SHELXL-2014/7 (Sheldrick, 2014)'

_computing_molecular_graphics ?

_computing_publication_material ?

_refine_special_details_?

_refine_ls_structure_factor_coef Fsqd

_refine_ls_matrix_type full

_refine_ls_weighting_scheme calc

_refine_ls_weighting_details

${ }^{\prime} \mathrm{w}=1 /\left[\backslash \mathrm{s}^{\wedge} 2^{\wedge}\left(\mathrm{Fo}^{\wedge} 2^{\wedge}\right)+(0.0727 \mathrm{P})^{\wedge} 2^{\wedge}\right]$ where $\mathrm{P}=\left(\mathrm{Fo}^{\wedge} 2^{\wedge}+2 \mathrm{Fc}^{\wedge} 2^{\wedge}\right) / 3^{\prime}$

_atom_sites_solution_primary ?

_atom_sites_solution_secondary ?

_atom_sites_solution_hydrogens geom

_refine_ls_hydrogen_treatment constr

_refine_ls_extinction_method none

_refine_ls_extinction_coef

_refine_ls_number_reflns 8910

_refine_1s_number_parameters 625

_refine_ls_number_restraints 62

_refine_ls_R_factor_all 0.0812

_refine_ls_R_factor_gt 0.0482

_refine_ls_wR_factor_ref 0.1265

_refine_ls_wR_factor_gt $\quad 0.1136$

_refine_ls_goodness_of_fit_ref 0.962

_refine_ls_restrained_S_all 0.959

_refine_ls_shift/su_max $\quad 0.000$

_refine_ls_shift/su_mean $\quad 0.000$

loop_ 
_atom_site_label

_atom_site_type_symbol

_atom_site_fract_x

_atom_site_fract_y

_atom_site_fract_z

_atom_site_U_iso_or_equiv

_atom_site_adp_type

_atom_site_occupancy

_atom_site_site_symmetry_order

_atom_site_calc_flag

_atom_site_refinement_flags_posn

_atom_site_refinement_flags_adp

_atom_site_refinement_flags_occupancy

_atom_site_disorder_assembly

atom_site_disorder_group

Fe Fe 0.41487(4) 0.41810(3) 0.20229(2) 0.04771(12) Uani $11 \mathrm{~d} \ldots \ldots$

$\mathrm{N} 1 \mathrm{~N} 0.5975(2)$ 0.50430(17) 0.24980(10) 0.0517(5) Uani $11 \mathrm{~d} \ldots \ldots$

C1 C 0.5796(3) 0.5817(2) 0.31517(13) 0.0622(7) Uani $11 \mathrm{~d} \ldots .$.

H1A H 0.56970 .66250 .30820 .075 Uiso 11 calc R U . .

H1B H 0.66140 .58790 .34880 .075 Uiso 11 calc R U . .

$\mathrm{N} 2 \mathrm{~N} 0.3527(2) 0.45851(18) 0.29188(11) 0.0572(5)$ Uani $11 \mathrm{~d} \ldots \ldots$

C4 C 0.4527(3) 0.5280(2) 0.34050(13) 0.0599(7) Uani $11 \mathrm{~d} \ldots .$.

C5 C 0.4356(5) 0.5514(3) 0.40862(16) 0.0831(10) Uani $11 \mathrm{~d} \ldots .$.

H5 H 0.50660 .59810 .44140 .100 Uiso 11 calc R U . .

C6 C 0.3126(6) 0.5050(4) 0.4271(2) 0.1037(14) Uani $11 \mathrm{~d}$. . . .

H6 H 0.29890 .52010 .47250 .124 Uiso 11 calc R U . .

C7 C 0.2100(5) 0.4361(4) 0.3780(2) 0.1008(13) Uani $11 \mathrm{~d} \ldots .$.

H7 H 0.12550 .40470 .38980 .121 Uiso 11 calc R U . . .

C8 C 0.2327(4) 0.4134(3) 0.31091(18) 0.0773(9) Uani $11 \mathrm{~d} \ldots .$.

H8 H 0.16290 .36560 .27790 .093 Uiso 11 calc R U . . .

C2 C 0.6911(3) 0.4145(2) 0.26116(15) 0.0631(7) Uani $11 \mathrm{~d} \ldots \ldots$ 
H2A H 0.75710 .44610 .30250 .076 Uiso 11 calc R U . .

H2B H 0.74350 .39960 .22330 .076 Uiso 11 calc R U . . .

N3 N 0.4739(2) 0.27653(18) 0.23287(11) 0.0568(5) Uani $11 \mathrm{~d} \ldots \ldots$

C9 C 0.6050(3) 0.2981(2) 0.26731(14) 0.0636(7) Uani $11 \mathrm{~d} \ldots .$.

C10 C 0.6557(4) 0.2181(3) 0.30372(18) 0.0882(10) Uani $11 \mathrm{~d} \ldots \ldots$

H10 H 0.74570 .23530 .32790 .106 Uiso 11 calc R U . .

C11 C 0.5699(6) 0.1115(3) 0.3035(2) 0.1035(14) Uani $11 \mathrm{~d} \ldots \ldots$

H11 H 0.60170 .05580 .32780 .124 Uiso 11 calc R U . .

C12 C 0.4394(5) 0.0881(3) 0.2681(2) 0.0930(12) Uani $11 \mathrm{~d} \ldots .$.

H12 H 0.38150 .01610 .26750 .112 Uiso 11 calc R U . . .

C13 C 0.3931(4) 0.1711(2) 0.23304(16) 0.0748(9) Uani $11 \mathrm{~d} \ldots \ldots$

H13 H 0.30330 .15430 .20870 .090 Uiso 11 calc R U . . .

C3 C 0.6352(3) 0.5753(2) 0.19613(14) 0.0590(7) Uani $11 \mathrm{~d} \ldots$.

H3 H 0.72620 .62750 .20930 .071 Uiso 11 calc R U . . .

N4 N 0.5174(2) 0.39486(19) 0.12367(11) 0.0552(5) Uani $11 \mathrm{~d} \ldots .$.

C14 C 0.6283(3) 0.4829(3) 0.13189(14) 0.0597(7) Uani $11 \mathrm{~d} \ldots$.

C15 C 0.7184(4) 0.4852(4) 0.0841(2) 0.0851(10) Uani $11 \mathrm{~d}$. . . .

H15 H 0.79420 .54690 .09030 .102 Uiso 11 calc R U . . .

C16 C 0.6928(5) 0.3932(4) 0.0266(2) 0.1020(13) Uani $11 \mathrm{~d}$. . . .

H16 H $0.75120 .3930-0.00670 .122$ Uiso 11 calc R U . .

C17 C 0.5827(5) 0.3035(4) 0.01896(18) 0.0916(11) Uani $11 \mathrm{~d} \ldots$.

H17 H $0.56580 .2409-0.01920 .110$ Uiso 11 calc R U . .

C18 C 0.4962(4) 0.3058(3) 0.06815(15) 0.0710(8) Uani $11 \mathrm{~d} \ldots \ldots$

H18 H 0.42090 .24390 .06280 .085 Uiso 11 calc R U . . .

N5 N 0.3915(2) 0.57706(18) 0.18252(10) 0.0510(5) Uani $11 \mathrm{~d} \ldots \ldots$

C19 C 0.5165(3) 0.6449(2) 0.18421(13) 0.0556(6) Uani $11 \mathrm{~d}$. . . .

C20 C 0.5281(4) 0.7607(2) 0.17154(15) 0.0681(8) Uani $11 \mathrm{~d} \ldots \ldots$

H20 H 0.61540 .80590 .17310 .082 Uiso 11 calc R U . . .

C21 C 0.4086(4) 0.8076(3) 0.15664(15) 0.0740(9) Uani $11 \mathrm{~d} \ldots \ldots$

H21 H 0.41380 .88480 .14700 .089 Uiso 11 calc R U . . .

C22 C 0.2816(4) 0.7402(3) 0.15607(15) 0.0711(8) Uani $11 \mathrm{~d} \ldots \ldots$ 
H22 H 0.19970 .77180 .14690 .085 Uiso 11 calc R U . .

C23 C 0.2757(3) 0.6253(2) 0.16911(13) 0.0598(7) Uani $11 \mathrm{~d} \ldots \ldots$

H23 H 0.18900 .57980 .16860 .072 Uiso 11 calc R U . .

C25A C 0.0186(7) 0.2447(11) 0.0661(3) 0.056(2) Uani 0.681(6) 1 d D . P A -1

C24A C 0.1379(10) 0.2991(10) 0.1134(5) 0.0536(19) Uani 0.681(6) 1 d D . P A -1

N6A N 0.2403(2) 0.33891(19) 0.15035(12) 0.0620(6) Uani 0.681(6) 1 d D . P A -1

C26A C 0.024(2) 0.262(3) -0.0014(6) 0.0866(18) Uani 0.681(6) $1 \mathrm{~d}$ D . P A -1

N7A N 0.030(2) 0.2797(11) -0.0556(4) 0.127(4) Uani 0.681(6) 1 d D . P A -1

C27A C -0.0903(10) 0.1694(12) 0.0848(3) 0.0598(11) Uani 0.681(6) 1 d D . P A -1

S1A S -0.0990(4) 0.1979(3) 0.1728(2) 0.0784(7) Uani 0.681(6) 1 d D . P A -1

C31 A C -0.1238(13) 0.0478(7) 0.1935(6) 0.112(3) Uani 0.681(6) 1 d D . P A -1

H24 H -0.2005 0.0414 0.2198 0.134 Uiso 0.681(6) 1 calc R U P A -1

H25 H -0.1487 -0.0127 0.1516 0.134 Uiso 0.681(6) 1 calc R U P A - 1

C32A C -0.0005(9) 0.0239(8) 0.2318(5) 0.145(3) Uani 0.681(6) 1 d D . P A -1

H26 H 0.02960 .08860 .27150 .174 Uiso 0.681(6) 1 calc R U P A -1

H27 H 0.07380 .02250 .20380 .174 Uiso 0.681(6) 1 calc R U P A -1

C33A C -0.0253(12) -0.0946(8) 0.2546(5) 0.165(4) Uani 0.681(6) 1 d D . P A -1

H28 H -0.1032 -0.0958 0.2796 0.248 Uiso 0.681(6) 1 calc R U P A -1

H29 H $0.0566-0.1040$ 0.2834 0.248 Uiso 0.681(6) 1 calc R U P A -1

H30 H -0.0451 -0.1597 0.2155 0.248 Uiso 0.681(6) 1 calc R U P A -1

C28A C -0.1965(15) 0.0910(17) 0.0393(3) 0.067(2) Uani 0.681(6) 1 d D . P A -1

C30A C -0.3231(7) 0.0475(7) 0.0613(6) 0.085(2) Uani 0.681(6) $1 \mathrm{~d}$ D . P A -1

N9A N -0.4223(16) 0.010(2) 0.0800(19) 0.136(5) Uani 0.681(6) $1 \mathrm{~d}$ D . P A -1

C29A C -0.1893(15) 0.0534(10)-0.0310(4) 0.074(2) Uani 0.681(6) 1 d D . P A -1

N8A N -0.193(2) 0.007(2) -0.0875(5) 0.106(3) Uani 0.681(6) 1 d D . P A -1

C25B C 0.0249(17) 0.224(3) 0.0688(6) 0.056(2) Uani 0.319(6) 1 d D . P A -2 C24B C 0.142(2) 0.277(3) 0.1179(11) 0.0536(19) Uani 0.319(6) 1 d D . P A -2 N6B N 0.2403(2) 0.33891(19) 0.15035(12) 0.0620(6) Uani 0.319(6) 1 d D . P A -2 C26B C 0.021(4) 0.265(6) 0.0065(14) 0.0866(18) Uani 0.319(6) 1 d D . P A -2 N7B N 0.026(5) 0.316(3) -0.0380(10) 0.127(4) Uani 0.319(6) 1 d D . P A -2 C27B C -0.092(2) 0.158(3) 0.0877(5) 0.0598(11) Uani 0.319(6) 1 d D . P A -2 
S1B S -0.0811(11) 0.1658(7) 0.1761(5) 0.0784(7) Uani 0.319(6) 1 d D . P A -2 C31B C -0.116(3) 0.0081(12) 0.1842(13) 0.112(3) Uani 0.319(6) 1 d D . P A -2 H31 H -0.2150 -0.0219 0.1709 0.134 Uiso 0.319(6) 1 calc R U P A -2 H32 H -0.0657 -0.0400 0.1535 0.134 Uiso 0.319(6) 1 calc R U P A -2 C32B C -0.0745(17) -0.0060(18) 0.2526(10) 0.145(3) Uani 0.319(6) 1 d D . P A -2 H33 H -0.1250 -0.0810 0.2596 0.174 Uiso 0.319(6) 1 calc R U P A -2 H34 H -0.0990 0.0601 0.2844 0.174 Uiso 0.319(6) 1 calc R U P A -2 C33B C 0.0797(18) -0.0076(18) 0.2669(11) 0.165(4) Uani 0.319(6) 1 d D . P A -2 H35 H 0.12950 .07230 .27010 .248 Uiso 0.319(6) 1 calc R U P A -2 H36 H $0.1072-0.06250 .23060 .248$ Uiso 0.319(6) 1 calc R U P A -2 H37 H 0.1009 -0.0335 0.3092 0.248 Uiso 0.319(6) 1 calc R U P A -2 C28B C -0.195(3) 0.076(4) 0.0426(7) 0.067(2) Uani 0.319(6) 1 d D . P A -2 C30B C -0.3127(16) 0.019(2) 0.0664(14) 0.085(2) Uani 0.319(6) 1 d D . P A -2 N9B N -0.417(4) -0.021(6) 0.080(4) 0.136(5) Uani 0.319(6) $1 \mathrm{~d}$ D . P A -2 C29B C -0.183(3) 0.032(3) -0.0266(9) 0.074(2) Uani 0.319(6) 1 d D . P A -2 N8B N -0.163(6) 0.022(6) -0.0829(12) 0.106(3) Uani 0.319(6) 1 d D . P A -2 C35A C 0.066(3) 0.711(3) 0.4785(8) 0.065(4) Uani 0.574(7) 1 d D . P B -3 C37A C 0.049(5) 0.762(5) 0.5462(13) 0.0844(12) Uani 0.574(7) 1 d D . P B -3 N11A N 0.028(5) 0.784(4) 0.6022(12) 0.111(5) Uani 0.574(7) 1 d D . P B -3 C36A C -0.0337(15) 0.6063(14) 0.4477(11) 0.060(3) Uani 0.574(7) 1 d D . P B -3 N10A N -0.134(3) 0.532(2) 0.4344(7) 0.083(2) Uani 0.574(7) 1 d D . P B -3 C34A C 0.162(2) 0.770(3) 0.4445(6) 0.0633(8) Uani 0.574(7) 1 d D . P B -3 S2A S 0.2943(14) 0.8784(7) 0.4981(6) 0.0818(11) Uani 0.574(7) 1 d D . P B -3 C41A C 0.286(3) 1.0110(12) 0.4629(10) 0.134(5) Uani 0.574(7) 1 d D . P B -3 H38 H 0.35651 .01630 .43310 .161 Uiso 0.574(7) 1 calc R U P B -3 H39 H 0.1955 1.0018 0.4350 0.161 Uiso 0.574(7) 1 calc R U P B -3 C42A C 0.3054(11) 1.1234(9) 0.5122(6) 0.145(3) Uani 0.574(7) 1 d D . P B -3 H40 H 0.2765 1.1856 0.4890 0.175 Uiso 0.574(7) 1 calc R U P B -3 H41 H 0.4045 1.1465 0.5290 0.175 Uiso 0.574(7) 1 calc R U P B -3 C43A C 0.226(4) 1.122(3) 0.5742(14) 0.232(8) Uani 0.574(7) 1 d D . P B -3 H42 H 0.13161 .08370 .55880 .348 Uiso 0.574(7) 1 calc R U P B -3 
H43 H 0.2274 1.2038 0.5976 0.348 Uiso 0.574(7) 1 calc R U P B -3 H44 H 0.27121 .07850 .60470 .348 Uiso 0.574(7) 1 calc R U P B -3 C38A C 0.1620(12) 0.7547(11) 0.3740(5) 0.055(2) Uani 0.574(7) 1 d D . P B -3 C39A C 0.2844(13) 0.7914(17) 0.3468(7) 0.058(3) Uani 0.574(7) 1 d D . P B -3 N12A N 0.387(2) 0.807(2) 0.3224(9) 0.073(3) Uani 0.574(7) 1 d D . P B -3 C40A C 0.0582(14) 0.6639(7) 0.3267(6) 0.067(2) Uani 0.574(7) 1 d D . P B -3 N13A N -0.0260(16) 0.6097(8) 0.2838(8) 0.100(3) Uani 0.574(7) 1 d D . P B -3 C35B C 0.048(4) 0.725(4) 0.4804(11) 0.065(4) Uani 0.426(7) 1 d D . P B -4 C37B C 0.049(7) 0.765(7) 0.5515(18) 0.0844(12) Uani 0.426(7) 1 d D . P B -4 N11B N 0.043(8) 0.814(5) 0.6063(17) 0.111(5) Uani 0.426(7) $1 \mathrm{~d}$ D . P B -4 C36B C -0.060(2) 0.627(2) 0.4497(16) 0.060(3) Uani 0.426(7) 1 d D . P B -4 N10B N -0.123(4) 0.534(3) 0.4177(10) 0.083(2) Uani 0.426(7) 1 d D . P B -4 C34B C 0.158(3) 0.767(3) 0.4481(8) 0.0633(8) Uani 0.426(7) 1 d D . P B -4 S2B S 0.303(2) 0.8571(9) 0.5039(9) 0.0818(11) Uani 0.426(7) $1 \mathrm{~d}$ D . P B -4 C41B C 0.314(4) 0.9995(17) 0.4778(15) 0.134(5) Uani 0.426(7) 1 d D . P B -4 H45 H 0.40451 .04690 .49740 .161 Uiso 0.426(7) 1 calc R U P B -4 H46 H 0.30940 .98500 .42850 .161 Uiso 0.426(7) 1 calc R U P B -4 C42B C 0.2067(14) 1.0699(12) 0.4967(9) 0.145(3) Uani 0.426(7) 1 d D . P B -4 H47 H 0.1171 1.0257 0.4731 0.175 Uiso 0.426(7) 1 calc R U P B -4 H48 H 0.22251 .14490 .48050 .175 Uiso 0.426(7) 1 calc R U P B -4 C43B C 0.197(7) 1.101(4) 0.5737(14) 0.232(8) Uani 0.426(7) 1 d D . P B -4 H49 H 0.21761 .03440 .59430 .348 Uiso 0.426(7) 1 calc R U P B -4 H49A H 0.1043 1.1159 0.5799 0.348 Uiso 0.426(7) 1 calc R U P B -4 H49B H 0.26311 .17190 .59480 .348 Uiso 0.426(7) 1 calc R U P B -4 C38B C 0.1753(16) 0.7306(16) 0.3796(7) 0.055(2) Uani 0.426(7) 1 d D . P B -4 C39B C 0.2998(18) 0.776(3) 0.3564(10) 0.058(3) Uani 0.426(7) 1 d D . P B -4 N12B N 0.395(3) 0.830(3) 0.3389(13) 0.073(3) Uani 0.426(7) 1 d D . P B -4 C40B C 0.0523(19) 0.7011(11) 0.3267(9) 0.067(2) Uani 0.426(7) 1 d D . P B -4 N13B N -0.043(2) 0.6562(12) 0.2877(12) 0.100(3) Uani 0.426(7) 1 d D . P B -4 loop_ 


$$
\begin{aligned}
& \text {-atom_site_aniso_label } \\
& \text {-atom_site_aniso_U_11 } \\
& \text {-atom_site_aniso_U_22 } \\
& \text {-atom_site_aniso_U_33 } \\
& \text {-atom_site_aniso_U_23 } \\
& \text {-atom_site_aniso_U_13 } \\
& \text { _atom_site_aniso_U_12 }
\end{aligned}
$$

Fe 0.0440(2) 0.04602(18) 0.0470(2) 0.00741(13) 0.00127(14) -0.00748(13)

N1 0.0477(12) 0.0509(11) 0.0520(12) 0.0110(9) -0.0011(9) -0.0020(9)

C1 0.0663(18) 0.0575(14) 0.0519(15) 0.0037(12) -0.0100(13) -0.0022(13)

N2 0.0595(14) 0.0530(11) 0.0604(13) 0.0166(10) 0.0130(11) 0.0028(10)

C4 0.078(2) 0.0509(13) 0.0510(15) 0.0116(11) 0.0081(13) 0.0122(13)

C5 0.124(3) 0.078(2) 0.0517(17) 0.0137(15) 0.0169(18) 0.028(2)

C6 0.158(4) 0.097(3) 0.078(3) 0.033(2) 0.054(3) 0.045(3)

C7 0.115(3) 0.095(3) 0.117(3) 0.048(3) 0.066(3) 0.025(2)

C8 0.080(2) 0.0705(18) 0.088(2) 0.0247(16) 0.0356(18) 0.0032(16)

C2 0.0551(17) 0.0676(16) 0.0610(16) 0.0105(13) -0.0061(13) 0.0061(13)

N3 0.0679(15) 0.0488(11) 0.0491(12) 0.0066(9) 0.0053(10) 0.0000(10)

C9 0.075(2) 0.0602(15) 0.0542(15) 0.0102(12) 0.0058(14) 0.0124(14)

C10 0.104(3) 0.090(2) 0.078(2) 0.0336(18) -0.0004(19) 0.028(2)

C11 0.160(5) 0.077(2) 0.090(3) 0.040(2) 0.023(3) 0.039(3)

C12 0.145(4) 0.0556(17) 0.082(2) 0.0213(16) 0.026(2) 0.007(2)

C13 0.101(3) 0.0493(14) 0.0697(19) 0.0113(13) 0.0153(17) -0.0067(15)

C3 0.0438(15) 0.0617(15) 0.0662(16) 0.0168(13) 0.0017(12) -0.0111(12)

N4 0.0538(13) 0.0599(12) 0.0493(12) 0.0106(10) 0.0028(10) 0.0045(10)

C14 0.0502(16) 0.0716(16) 0.0591(16) 0.0201(13) 0.0095(12) 0.0046(12)

C15 0.067(2) 0.106(3) 0.094(3) 0.039(2) 0.0302(19) 0.0129(18)

C16 0.116(3) 0.133(3) 0.077(2) 0.034(2) 0.048(2) 0.045(3)

C17 0.110(3) 0.106(3) 0.064(2) 0.0115(18) 0.025(2) 0.030(2)

C18 0.081(2) 0.0733(18) 0.0550(16) 0.0050(14) 0.0027(15) 0.0149(15)

N5 0.0474(13) 0.0529(11) 0.0479(11) 0.0096(9) -0.0007(9) -0.0025(9) 
C19 0.0555(16) 0.0548(13) 0.0518(14) 0.0130(11) 0.0023(12) -0.0063(12)

C20 0.079(2) 0.0557(15) 0.0658(18) 0.0177(13) 0.0086(15) -0.0083(14)

C21 0.105(3) 0.0580(16) 0.0603(18) 0.0176(13) 0.0111(17) 0.0111(17)

C22 0.088(2) 0.0727(18) 0.0553(17) 0.0146(14) 0.0003(15) 0.0283(17)

C23 0.0568(17) 0.0667(16) 0.0528(15) 0.0088(12)0.0027(12) 0.0069(13)

C25A 0.0434(16) 0.047(5) 0.0719(19) 0.0141(15) -0.0059(13) -0.003(2)

C24A 0.0507(17) 0.039(5) 0.067(2) 0.012(2) 0.0030(14) -0.003(2)

N6A 0.0543(14) 0.0594(12) 0.0635(14) 0.0068(10) 0.0027(11) -0.0103(11)

C26A 0.063(2) 0.100(3) 0.082(4) 0.027(4) -0.011(3) -0.030(2)

N7A 0.120(3) 0.152(9) 0.079(5) 0.031(6) -0.021(6) -0.065(7)

C27A 0.0469(16) 0.052(3) 0.0770(19) 0.0173(14) -0.0031(14) -0.0002(14)

S1A 0.0670(13) 0.0794(18) 0.0872(8) 0.0242(12) 0.0089(8) -0.0023(12)

C31A 0.096(4) 0.117(6) 0.128(5) 0.072(5) -0.011(3) -0.007(5)

C32A 0.127(8) 0.163(7) 0.160(8) 0.087(5) 0.007(6) 0.017(6)

C33A 0.195(10) 0.127(6) 0.180(8) 0.071(6) -0.019(7) 0.033(6)

C28A 0.0507(17) 0.053(5) 0.091(2) 0.026(2) -0.0120(16) -0.007(2)

C30A 0.061(2) 0.075(5) 0.118(4) 0.062(3) -0.028(2) -0.021(3)

N9A 0.076(3) 0.161(15) 0.168(4) 0.104(10) -0.028(3) -0.051(5)

C29A 0.068(3) 0.043(5) 0.099(3) 0.015(2) -0.026(2) 0.000(3)

N8A 0.101(10) 0.084(7) 0.104(3) -0.011(3) -0.034(4) 0.002(6)

C25B 0.0434(16) 0.047(5) 0.0719(19) 0.0141(15) -0.0059(13) -0.003(2)

C24B 0.0507(17) 0.039(5) 0.067(2) 0.012(2) 0.0030(14) -0.003(2)

N6B 0.0543(14) 0.0594(12) 0.0635(14) 0.0068(10) 0.0027(11) -0.0103(11)

C26B 0.063(2) 0.100(3) 0.082(4) 0.027(4) -0.011(3) -0.030(2)

N7B 0.120(3) 0.152(9) 0.079(5) 0.031(6) -0.021(6) -0.065(7)

C27B 0.0469(16) 0.052(3) 0.0770(19) 0.0173(14) -0.0031(14) -0.0002(14)

S1B 0.0670(13) 0.0794(18) 0.0872(8) 0.0242(12) 0.0089(8) -0.0023(12)

C31B 0.096(4) 0.117(6) 0.128(5) 0.072(5) -0.011(3) -0.007(5)

C32B 0.127(8) 0.163(7) 0.160(8) 0.087(5) 0.007(6) 0.017(6)

C33B 0.195(10) 0.127(6) 0.180(8) 0.071(6) -0.019(7) 0.033(6)

C28B 0.0507(17) 0.053(5) 0.091(2) 0.026(2) -0.0120(16) -0.007(2) 
C30B 0.061(2) 0.075(5) 0.118(4) 0.062(3) -0.028(2) -0.021(3)

N9B 0.076(3) 0.161(15) 0.168(4) 0.104(10) -0.028(3) -0.051(5)

C29B 0.068(3) 0.043(5) 0.099(3) 0.015(2) -0.026(2) 0.000(3)

N8B 0.101(10) 0.084(7) 0.104(3) -0.011(3) -0.034(4) 0.002(6)

C35A 0.048(6) 0.072(7) 0.071(2) 0.012(2) 0.0014(19) 0.007(6)

C37A 0.065(2) 0.097(4) 0.081(4) 0.009(4) 0.004(4) -0.004(2)

N11A 0.095(10) 0.124(19) 0.088(4) -0.016(5) 0.015(5) -0.024(10)

C36A 0.045(5) 0.074(5) 0.064(2) 0.019(4) 0.003(4) 0.012(5)

N10A 0.079(4) 0.111(2) 0.051(7) 0.026(5) 0.006(6) -0.028(2)

C34A 0.0530(19) 0.0630(17) 0.072(2) 0.0177(18) -0.0007(16) 0.0063(13)

S2A 0.0715(16) 0.078(2) 0.0819(19) 0.005(2) -0.0036(11) -0.012(2)

C41A 0.153(11) 0.076(3) 0.156(10) 0.015(3) 0.006(9) -0.017(4)

C42A 0.090(6) 0.121(6) 0.201(9) -0.023(6) 0.007(6) 0.018(4)

C43A 0.22(2) 0.119(11) 0.313(13) -0.037(7) -0.053(10) 0.046(14)

C38A 0.054(3) 0.046(5) 0.066(2) 0.028(2) -0.0090(18) 0.009(3)

C39A 0.069(3) 0.053(5) 0.054(4) 0.027(3) -0.007(3) 0.013(3)

N12A 0.085(3) 0.070(8) 0.058(8) 0.004(5) 0.010(5) -0.001(3)

C40A 0.077(3) 0.048(6) 0.073(2) 0.029(4) -0.0067(18) -0.007(4)

N13A 0.102(5) 0.090(7) 0.091(3) 0.030(6) -0.026(3) -0.028(6)

C35B 0.048(6) 0.072(7) 0.071(2) 0.012(2) 0.0014(19) 0.007(6)

C37B 0.065(2) 0.097(4) 0.081(4) 0.009(4) 0.004(4) -0.004(2)

N11B 0.095(10) 0.124(19) 0.088(4) -0.016(5) 0.015(5) -0.024(10)

C36B 0.045(5) 0.074(5) 0.064(2) 0.019(4) 0.003(4) 0.012(5)

N10B 0.079(4) 0.111(2) 0.051(7) 0.026(5) 0.006(6)-0.028(2)

C34B 0.0530(19) 0.0630(17) 0.072(2) 0.0177(18) -0.0007(16) 0.0063(13)

S2B 0.0715(16) 0.078(2) 0.0819(19) 0.005(2) -0.0036(11) -0.012(2)

C41B 0.153(11) 0.076(3) 0.156(10) 0.015(3) 0.006(9) -0.017(4)

C42B 0.090(6) 0.121(6) 0.201(9) -0.023(6) 0.007(6) 0.018(4)

C43B 0.22(2) 0.119(11) 0.313(13) -0.037(7) -0.053(10) 0.046(14)

C38B 0.054(3) 0.046(5) 0.066(2) 0.028(2) -0.0090(18) 0.009(3)

C39B 0.069(3) 0.053(5) 0.054(4) 0.027(3) -0.007(3) 0.013(3) 
N12B 0.085(3) 0.070(8) 0.058(8) 0.004(5) 0.010(5) -0.001(3)

C40B 0.077(3) 0.048(6) 0.073(2) 0.029(4) -0.0067(18) -0.007(4)

N13B 0.102(5) 0.090(7) 0.091(3) 0.030(6) -0.026(3) -0.028(6)

_geom_special_details

;

All esds (except the esd in the dihedral angle between two 1.s. planes) are estimated using the full covariance matrix. The cell esds are taken into account individually in the estimation of esds in distances, angles and torsion angles; correlations between esds in cell parameters are only used when they are defined by crystal symmetry. An approximate (isotropic) treatment of cell esds is used for estimating esds involving 1.s. planes.

;

loop_

_geom_bond_atom_site_label_1

_geom_bond_atom_site_label_2

_geom_bond_distance

_geom_bond_site_symmetry_2

_geom_bond_publ_flag

Fe N6B 1.907(2) . ?

Fe N6A 1.907(2) . ?

Fe N3 1.957(2) . ?

Fe N5 1.958(2) . ?

Fe N1 1.962(2) . ?

Fe N2 1.967(2) . ?

Fe N4 1.968(2) . ?

N1 C2 1.487(4) . ?

N1 C1 1.488(3) . ?

N1 C3 1.514(3) . ?

C1 C4 1.494(4) . ? 
C1 H1A 0.9700 .?

C1 H1B 0.9700 . ?

N2 C8 1.342(4) . ?

N2 C4 1.351(3) .?

C4 C5 1.384(4) . ?

C5 C6 1.367(6) . ?

C5 H5 0.9300 .?

C6 C7 1.367(6) . ?

C6 H6 0.9300 . ?

C7 C8 1.379(5) . ?

C7 H7 0.9300. ?

C8 H8 0.9300 . ?

C2 C9 1.506(4) . ?

C2 H2A 0.9700 . ?

C2 H2B 0.9700 . ?

N3 C13 1.347(3) . ?

N3 C9 1.351(4) . ?

C9 C10 1.374(4) . ?

C10 C11 1.380(5).?

C10 H10 0.9300 . ?

C11 C12 1.351(6) . ?

C11 H11 0.9300.?

C12 C13 1.369(5).?

C12 H12 0.9300 .?

C13 H13 0.9300.?

C3 C14 1.500(4) . ?

C3 C19 1.507(4) . ?

C3 H3 0.9800. ?

N4 C18 1.340(4) . ?

N4 C14 1.347(3) . ?

C14 C15 1.379(4) . ? 
C15 C16 1.387(6) . ?

C15 H15 0.9300 .?

C16 C17 1.355(6) . ?

C16 H16 0.9300.?

C17 C18 1.375(5) . ?

C17 H17 0.9300 . ?

C18 H18 0.9300.?

N5 C23 1.339(4) . ?

N5 C19 1.349(3) . ?

C19 C20 1.380(4) . ?

C20 C21 1.369(5). ?

C20 H20 0.9300 . ?

C21 C22 1.367(5).?

C21 H21 0.9300 .?

C22 C23 1.376(4) .?

C22 H22 0.9300 .?

C23 H23 0.9300.?

C25A C24A 1.407(4). ?

C25A C27A 1.407(4) . ?

C25A C26A 1.415(7) . ?

C24A N6A 1.157(4) . ?

C26A N7A 1.154(8) . ?

C27A C28A 1.393(4) . ?

C27A S1A 1.757(4). ?

S1A C31A 1.825(5). ?

C31A C32A 1.435(11).?

C31A H24 0.9700 . ?

C31A H25 0.9700 . ?

C32A C33A 1.501(9) . ?

C32A H26 0.9700 .?

C32A H27 0.9700 . ? 
C33A H28 0.9600 .?

C33A H29 0.9600 . ?

C33A H30 0.9600 . ?

C28A C29A 1.416(6). ?

C28A C30A 1.420(6).?

C30A N9A 1.140(5) . ?

C29A N8A 1.160(8) . ?

C25B C27B 1.408(5).?

C25B C24B 1.410(5) .?

C25B C26B 1.417(7).?

C24B N6B 1.160(5) . ?

C26B N7B 1.155(10) . ?

C27B C28B 1.393(5) . ?

C27B S1B 1.757(5) . ?

S1B C31B 1.823(6) . ?

C31B C32B 1.433(12) . ?

C31B H31 0.9700 . ?

C31B H32 0.9700 . ?

C32B C33B 1.501(10) . ?

C32B H33 0.9700 . ?

C32B H34 0.9700 . ?

C33B H35 0.9600 . ?

C33B H36 0.9600 . ?

C33B H37 0.9600 . ?

C28B C29B 1.418(6) .?

C28B C30B 1.421(7) . ?

C30B N9B 1.141(6) . ?

C29B N8B 1.161(9) . ?

C35A C34A 1.398(7) . ?

C35A C37A 1.420(10) . ?

C35A C36A 1.424(5) . ? 
C37A N11A 1.157(10) . ?

C36A N10A 1.180(9) . ?

C34A C38A 1.399(6) . ?

C34A S2A 1.768(5) . ?

S2A C41A 1.787(6) . ?

C41A C42A 1.445(17) . ?

C41A H38 0.9700 . ?

C41A H39 0.9700 . ?

C42A C43A 1.545(8) . ?

C42A H40 0.9700 .?

C42A H41 0.9700 . ?

C43A H42 0.9600 . ?

C43A H43 0.9600 .?

C43A H44 0.9600 . ?

C38A C39A 1.417(5) . ?

C38A C40A 1.463(6) . ?

C39A N12A 1.171(8) . ?

C40A N13A 1.143(5). ?

C35B C34B 1.398(7) . ?

C35B C37B 1.420(11).?

C35B C36B 1.423(5) . ?

C37B N11B 1.157(11) . ?

C36B N10B 1.180(10) . ?

C34B C38B 1.403(8) . ?

C34B S2B 1.769(5) . ?

S2B C41B 1.787(6) . ?

C41B C42B 1.445(17) . ?

C41B H45 0.9700 . ?

C41B H46 0.9700 . ?

C42B C43B 1.544(7) . ?

C42B H47 0.9700 . ? 
C42B H48 0.9700 . ?

C43B H49 0.9600 . ?

C43B H49A 0.9600 . ?

C43B H49B 0.9600 . ?

C38B C39B 1.421(5) . ?

C38B C40B 1.466(7) . ?

C39B N12B 1.171(9) . ?

C40B N13B 1.143(5) . ?

loop_

_geom_angle_atom_site_label_1

_geom_angle_atom_site_label_2

_geom_angle_atom_site_label_3

_geom_angle

_geom_angle_site_symmetry_1

_geom_angle_site_symmetry_3

_geom_angle_publ_flag

N6B Fe N3 97.32(10) . . ?

N6A Fe N3 97.32(10) . . ?

N6B Fe N5 94.24(9) . . ?

N6A Fe N5 94.24(9) . . ?

N3 Fe N5 168.43(9) . . ?

N6B Fe N1 175.79(9) . . ?

N6A Fe N1 175.79(9) . . ?

N3 Fe N1 85.32(9) . . ?

N5 Fe N1 83.15(8) . . ?

N6B Fe N2 98.64(10) . . ?

N6A Fe N2 98.64(10) . . ?

N3 Fe N2 86.13(9) . . ?

N5 Fe N2 91.99(9) . . ?

N1 Fe N2 84.78(9) . . ? 
N6B Fe N4 93.93(9) . . ?

N6A Fe N4 93.93(9) . . ?

N3 Fe N4 92.14(9) . . ?

N5 Fe N4 87.21(9) . . ?

N1 Fe N4 82.66(9) . . ?

N2 Fe N4 167.42(9) . . ?

C2 N1 C1 111.7(2) . . ?

C2 N1 C3 113.9(2) . . ?

C1 N1 C3 113.6(2) . ?

C2 N1 Fe 109.03(15) ..?

C1 N1 Fe 109.62(16) ..?

C3 N1 Fe 98.04(14) . . ?

N1 C1 C4 109.7(2) . . ?

N1 C1 H1A 109.7 . . ?

C4 C1 H1A 109.7 . .?

N1 C1 H1B 109.7 . . ?

C4 C1 H1B 109.7 . . ?

H1A C1 H1B 108.2 . . ?

C8 N2 C4 118.2(3) . . ?

C8 N2 Fe 127.6(2) . . ?

C4 N2 Fe 113.55(18) ..?

N2 C4 C5 122.0(3) . . ?

N2 C4 C1 115.4(2) . . ?

C5 C4 C1 122.6(3) . . ?

C6 C5 C4 119.0(4) . . ?

C6 C5 H5 120.5 .. ?

C4 C5 H5 120.5 ..?

C5 C6 C7 119.4(3) . . ?

C5 C6 H6 120.3 .. ?

C7 C6 H6 120.3 . . ?

C6 C7 C8 119.6(4) . . ? 
C6 C7 H7 120.2 . . ?

C8 C7 H7 120.2 . . ?

N2 C8 C7 121.9(4) . . ?

N2 C8 H8 119.1 . .?

C7 C8 H8 119.1 .. ?

N1 C2 C9 109.5(2) . . ?

N1 C2 H2A 109.8 . ?

C9 C2 H2A 109.8 . . ?

N1 C2 H2B 109.8 . . ?

C9 C2 H2B 109.8 . . ?

H2A C2 H2B 108.2 . .?

C13 N3 C9 118.2(3) . . ?

C13 N3 Fe 127.3(2) .. ?

C9 N3 Fe 113.54(17) .. ?

N3 C9 C10 122.0(3) . . ?

N3 C9 C2 114.8(2) . . ?

C10 C9 C2 123.2(3) . . ?

C9 C10 C11 118.4(4) . . ?

C9 C10 H10 120.8 . . ?

C11 C10 H10 120.8 . . ?

C12 C11 C10 119.9(3) .. ?

C12 C11 H11 120.0 . ?

C10 C11 H11 120.0 . .?

C11 C12 C13 119.6(3) . . ?

C11 C12 H12 120.2 . ?

C13 C12 H12 120.2 ..?

N3 C13 C12 121.9(4) . . ?

N3 C13 H13 119.1 ..?

C12 C13 H13 119.1 . . ?

C14 C3 C19 106.3(2) . . ?

C14 C3 N1 105.7(2)..? 
C19 C3 N1 105.6(2).. ?

C14 C3 H3 112.9 . . ?

C19 C3 H3 112.9 . . ?

N1 C3 H3 112.9 . . ?

C18 N4 C14 118.8(3) ..?

C18 N4 Fe 130.3(2) .. ?

C14 N4 Fe 110.85(17). . ?

N4 C14 C15 121.8(3) . . ?

N4 C14 C3 111.7(2) . . ?

C15 C14 C3 126.5(3) .. ?

C14 C15 C16 118.2(3) .. ?

C14 C15 H15 120.9 . . ?

C16 C15 H15 120.9 . . ?

C17 C16 C15 119.9(3) .. ?

C17 C16 H16 120.1 .. ?

C15 C16 H16 120.1 .. ?

C16 C17 C18 119.4(4) . . ?

C16 C17 H17 120.3 ..?

C18 C17 H17 120.3 .. ?

N4 C18 C17 121.8(3) . ?

N4 C18 H18 119.1 . . ?

C17 C18 H18 119.1 ..?

C23 N5 C19 118.4(2) . . ?

C23 N5 Fe 130.72(17). . ?

C19 N5 Fe 110.90(18). . ?

N5 C19 C20 122.1(3) . . ?

N5 C19 C3 111.7(2) . . ?

C20 C19 C3 126.1(2).. ?

C21 C20 C19 118.7(3) .. ?

C21 C20 H20 120.7 . . ?

C19 C20 H20 120.7 . . ? 
C22 C21 C20 119.5(3) . . ?

C22 C21 H21 120.2 . ?

C20 C21 H21 120.2 . .?

C21 C22 C23 119.5(3)..?

C21 C22 H22 120.3 . .?

C23 C22 H22 120.3 ..?

N5 C23 C22 121.8(3). . ?

N5 C23 H23 119.1 ..?

C22 C23 H23 119.1 . .?

C24A C25A C27A 120.9(3) . . ?

C24A C25A C26A 115.1(4) . . ?

C27A C25A C26A 123.7(4) . . ?

N6A C24A C25A 175.9(17) . . ?

C24A N6A Fe 172.0(5) . . ?

N7A C26A C25A 178(2) . . ?

C28A C27A C25A 125.1(4) . . ?

C28A C27A S1A 120.4(3) . . ?

C25A C27A S1A 113.8(3). . ?

C27A S1A C31A 104.1(3) . . ?

C32A C31A S1A 112.0(6) . . ?

C32A C31A H24 109.2 . ?

S1A C31A H24 109.2 . . ?

C32A C31A H25 109.2 . . ?

S1A C31A H25 109.2 . ?

H24 C31A H25 107.9 . . ?

C31A C32A C33A 111.9(8) . . ?

C31A C32A H26 109.2 . . ?

C33A C32A H26 109.2 . . ?

C31A C32A H27 109.2 . ?

C33A C32A H27 109.2 . ?

H26 C32A H27 107.9 . . ? 
C32A C33A H28 109.5 . ?

C32A C33A H29 109.5 . . ?

H28 C33A H29 109.5 . ?

C32A C33A H30 109.5 . . ?

H28 C33A H30 109.5 . . ?

H29 C33A H30 109.5 . . ?

C27A C28A C29A 123.5(4) . . ?

C27A C28A C30A 120.8(4) . . ?

C29A C28A C30A 115.7(4) . . ?

N9A C30A C28A 177.5(15) . . ?

N8A C29A C28A 170.2(14) . . ?

C27B C25B C24B 120.3(7) . . ?

C27B C25B C26B 123.3(8) . . ?

C24B C25B C26B 114.6(7) . . ?

N6B C24B C25B 166(2) . . ?

C24B N6B Fe 171.1(19) .. ?

N7B C26B C25B 170(4) . . ?

C28B C27B C25B 124.9(6) . . ?

C28B C27B S1B 120.4(6) . . ?

C25B C27B S1B 113.6(6) . . ?

C27B S1B C31B 104.1(5) .. ?

C32B C31B S1B 111.7(9) . . ?

C32B C31B H31 109.3 . . ?

S1B C31B H31 109.3 . . ?

C32B C31B H32 109.3 . ?

S1B C31B H32 109.3 . . ?

H31 C31B H32 107.9 . . ?

C31B C32B C33B 111.7(11) . ?

C31B C32B H33 109.3 . . ?

C33B C32B H33 109.3 . . ?

C31B C32B H34 109.3 . . ? 
C33B C32B H34 109.3 . . ?

H33 C32B H34 107.9 . . ?

С32B C33B H35 109.5 . . ?

C32B C33B H36 109.5 . . ?

H35 C33B H36 109.5 . . ?

C32B C33В H37 109.5 . . ?

H35 C33B H37 109.5 . . ?

H36 C33B H37 109.5 . . ?

C27B C28B C29B 123.3(6) . . ?

C27B C28B C30B 120.7(7) . . ?

C29B C28B C30B 115.5(7) . . ?

N9B C30B C28B 171(4) . . ?

N8B C29B C28B 165(4).. ?

C34A C35A C37A 121.0(8) . . ?

C34A C35A C36A 124.7(5) . . ?

C37A C35A C36A 113.8(4) . . ?

N11A C37A C35A 169(5) . . ?

N10A C36A C35A 163.6(16) . . ?

C35A C34A C38A 127.5(4) . . ?

C35A C34A S2A 114.9(4) . . ?

C38A C34A S2A 117.5(5) . . ?

C34A S2A C41A 103.5(3) . . ?

C42A C41A S2A 115.3(11) . . ?

C42A C41A H38 108.4 . ?

S2A C41A H38 108.4 . . ?

C42A C41A H39 108.4 . . ?

S2A C41A H39 108.4 . . ?

H38 C41A H39 107.5 . . ?

C41A C42A C43A 116.0(15) . . ?

C41A C42A H40 108.3 . ?

C43A C42A H40 108.3 . . ? 
C41A C42A H41 108.3 . . ?

C43A C42A H41 108.3 . . ?

H40 C42A H41 107.4 . . ?

C42A C43A H42 109.5 . . ?

C42A C43A H43 109.5 . . ?

H42 C43A H43 109.5 . . ?

C42A C43A H44 109.5 . . ?

H42 C43A H44 109.5 . . ?

H43 C43A H44 109.5 . . ?

C34A C38A C39A 121.1(4) . ?

C34A C38A C40A 120.5(7) . . ?

C39A C38A C40A 114.6(5) . . ?

N12A C39A C38A 172(2) . . ?

N13A C40A C38A 167.7(9) . . ?

C34B C35B C37B 120.9(10) . . ?

C34B C35B C36B 124.6(6) . . ?

C37B C35B C36B 113.8(6) . . ?

N11B C37B C35B 169(9) . . ?

N10B C36B C35B 164(3) . . ?

C35B C34B C38B 127.0(6) . . ?

C35B C34B S2B 114.8(5) . . ?

C38B C34B S2B 117.3(8) . . ?

C34B S2B C41B 103.5(4) . . ?

C42B C41B S2B 115.0(12) . . ?

C42B C41B H45 108.5 . . ?

S2B C41B H45 108.5 . . ?

C42B C41B H46 108.5 . . ?

S2B C41B H46 108.5 . . ?

H45 C41B H46 107.5 . . ?

C41B C42B C43B 116.0(15) . . ?

C41B C42B H47 108.3 . ? 
C43B C42B H47 108.3 . . ?

C41B C42B H48 108.3 . ?

C43B C42B H48 108.3 . . ?

H47 C42B H48 107.4 . . ?

C42B C43B H49 109.5 . . ?

C42B C43B H49A 109.5 . . ?

H49 C43B H49A 109.5 . . ?

C42B C43B H49B 109.5 . . ?

H49 C43B H49B 109.5 . . ?

H49A C43B H49B 109.5 . . ?

C34B C38B C39B 119.8(6) . . ?

C34B C38B C40B 119.5(10) . . ?

C39B C38B C40B 113.7(7) . . ?

N12B C39B C38B 170(3) . . ?

N13B C40B C38B 166.9(14) . . ?

loop_

_geom_torsion_atom_site_label_1

_geom_torsion_atom_site_label_2

_geom_torsion_atom_site_label_3

_geom_torsion_atom_site_label_4

_geom_torsion

_geom_torsion_site_symmetry_1

_geom_torsion_site_symmetry_2

_geom_torsion_site_symmetry_3

_geom_torsion_site_symmetry_4

_geom_torsion_publ_flag

C2 N1 C1 C4 -93.1(3)....?

C3 N1 C1 C4 136.4(2).... ?

Fe N1 C1 C4 27.8(3)... . ?

C8 N2 C4 C5 1.2(4)....? 
Fe N2 C4 C5 -170.7(2) ... . ?

C8 N2 C4 C1 -176.1(3) .... ?

Fe N2 C4 C1 12.0(3) ... . ?

$\mathrm{N} 1 \mathrm{C} 1 \mathrm{C} 4 \mathrm{~N} 2-26.5(3) \ldots$ ?

N1 C1 C4 C5 156.2(3) ... . ?

N2 C4 C5 C6 -1.3(5) ....?

C1 C4 C5 C6 175.8(3).... ?

C4 C5 C6 C7 0.4(6) .... ?

C5 C6 C7 C8 0.7(6) ....?

C4 N2 C8 C7 -0.1(5) ....?

Fe N2 C8 C7 170.5(3) . . . ?

C6 C7 C8 N2 -0.8(6) ... ? ?

C1 N1 C2 C9 92.3(2).... ?

C3 N1 C2 C9 -137.3(2).... ?

Fe N1 C2 C9 -28.9(3) ... . ?

C13 N3 C9 C10 -2.1(4).... ?

Fe N3 C9 C10 167.5(3) . . . . ?

C13 N3 C9 C2 176.6(3).... ?

Fe N3 C9 C2 -13.9(3) .... ?

N1 C2 C9 N3 28.6(3).... ?

N1 C2 C9 C10 -152.8(3).... ?

N3 C9 C10 C11 1.4(5) ... . ?

C2 C9 C10 C11 -177.2(3).... ?

C9 C10 C11 C12 0.0(6) ... . ?

C10 C11 C12 C13 -0.5(6)....?

C9 N3 C13 C12 1.5(5).... ?

Fe N3 C13 C12 -166.5(3) .... ?

C11 C12 C13 N3 -0.2(6)... . ?

C2 N1 C3 C14 58.6(3) ... . ?

C1 N1 C3 C14-172.0(2).... ?

Fe N1 C3 C14 -56.5(2) . . . ? 
C2 N1 C3 C19 171.02(19) ... . ?

C1 N1 C3 C19-59.6(3)... . ?

Fe N1 C3 C19 56.00(19) . . . . ?

C18 N4 C14 C15 1.5(4) ... . ?

Fe N4 C14 C15 178.7(3) ... . ?

C18 N4 C14 C3 179.5(3) ... . ?

Fe N4 C14 C3 -3.3(3) ....?

C19 C3 C14 N4 -70.8(3).... ?

N1 C3 C14 N4 41.1(3) ... . ?

C19 C3 C14 C15 107.1(3)....?

N1 C3 C14 C15 -141.0(3)....?

N4 C14 C15 C16 -0.4(5) . . . ?

C3 C14 C15 C16-178.1(3) ... . ?

C14 C15 C16 C17 -0.8(6) . . . ?

C15 C16 C17 C18 0.9(6) . . . ?

C14 N4 C18 C17 -1.3(5) ... . ?

Fe N4 C18 C17 -178.0(2) ... . ?

C16 C17 C18 N4 0.2(6)... . ?

C23 N5 C19 C20 -1.1(4).... ?

Fe N5 C19 C20 179.6(2) ... . ?

C23 N5 C19 C3 -176.9(2).... ?

Fe N5 C19 C3 3.7(3) ... . ?

C14 C3 C19 N5 70.7(3)... . ?

N1 C3 C19 N5 -41.3(3)....?

C14 C3 C19 C20 -104.9(3) ... . ?

N1 C3 C19 C20 143.0(3) ... . ?

N5 C19 C20 C21 -0.2(4) ... . ?

C3 C19 C20 C21 175.1(3)... . ?

C19 C20 C21 C22 1.4(4)... . ?

C20 C21 C22 C23 -1.3(5).... ?

C19 N5 C23 C22 1.1(4)....? 
Fe N5 C23 C22 -179.7(2) ... . ?

C21 C22 C23 N5 0.1(4)...?

C24A C25A C27A C28A -165.1(17) ....?

C26A C25A C27A C28A 8(3) ... . ?

C24A C25A C27A S1A 24(2) ... . ?

C26A C25A C27A S1A -162.2(18) .... ?

C28A C27A S1A C31A 53.9(15) ... . ?

C25A C27A S1A C31A -134.9(13) ....?

C27A S1A C31A C32A 107.7(12) ... ?

S1A C31A C32A C33A 174.5(9) ... . ?

C25A C27A C28A C29A 17(3) .... ?

S1A C27A C28A C29A -172.8(15) ... . ?

C25A C27A C28A C30A -160.3(16) .... ?

S1A C27A C28A C30A 10(2) .... ?

C27B C25B C24B N6B -147(14) .... ?

C26B C25B C24B N6B 19(17) ... . ?

C27B C25B C26B N7B 126(31)....?

C24B C25B C26B N7B -39(32) ... . ?

C24B C25B C27B C28B -162(4) .... ?

C26B C25B C27B C28B 34(6) .... ?

C24B C25B C27B S1B 6(5) ... . ?

C26B C25B C27B S1B -158(4) ....?

C28B C27B S1B C31B 40(3) .... ?

C25B C27B S1B C31B -128(3) ... . ?

C27B S1B C31B C32B 164(2) ... . ?

S1B C31B C32B C33B -81(2) ... . ?

C25B C27B C28B C29B 12(7) .... ?

S1B C27B C28B C29B -156(4) .... ?

C25B C27B C28B C30B -177(4) .... ?

S1B C27B C28B C30B 15(6) .... ?

C27B C28B C29B N8B -47(18) ... . ? 
C30B C28B C29B N8B 142(16) ... . ?

C34A C35A C36A N10A -152(8) .... ?

C37A C35A C36A N10A 20(12) ... . ?

C37A C35A C34A C38A -158(5) .... ?

C36A C35A C34A C38A 13(6) .... ?

C37A C35A C34A S2A 20(5) ... . ?

C36A C35A C34A S2A -169(3) ... . ?

C35A C34A S2A C41A -128(3) ... . ?

C38A C34A S2A C41A 50(2) ... . ?

C34A S2A C41A C42A 141(2) ... . ?

S2A C41A C42A C43A -44(3).... ?

C35A C34A C38A C39A -161(3) .... ?

S2A C34A C38A C39A 21(3) ... . ?

C35A C34A C38A C40A -4(4) ....?

S2A C34A C38A C40A 178.2(15) ....?

C34A C38A C40A N13A 131(8) .... ?

C39A C38A C40A N13A -70(8) .... ?

C34B C35B C37B N11B 79(25) . . . ? ?

C36B C35B C37B N11B -111(20) ... . ?

C34B C35B C36B N10B 38(17) ... . ?

C37B C35B C36B N10B -132(12) ... ?

C37B C35B C34B C38B 176(6) ... . ?

C36B C35B C34B C38B 7(8) ... . ?

C37B C35B C34B S2B 8(7) ... . ?

C36B C35B C34B S2B -162(4) .... ?

C35B C34B S2B C41B -119(4) .... ?

C38B C34B S2B C41B 71(3) .... ?

C34B S2B C41B C42B 73(3) .... ?

S2B C41B C42B C43B 58(4) .... ?

C35B C34B C38B C39B -175(4) .... ?

S2B C34B C38B C39B -6(4) ... . ? 


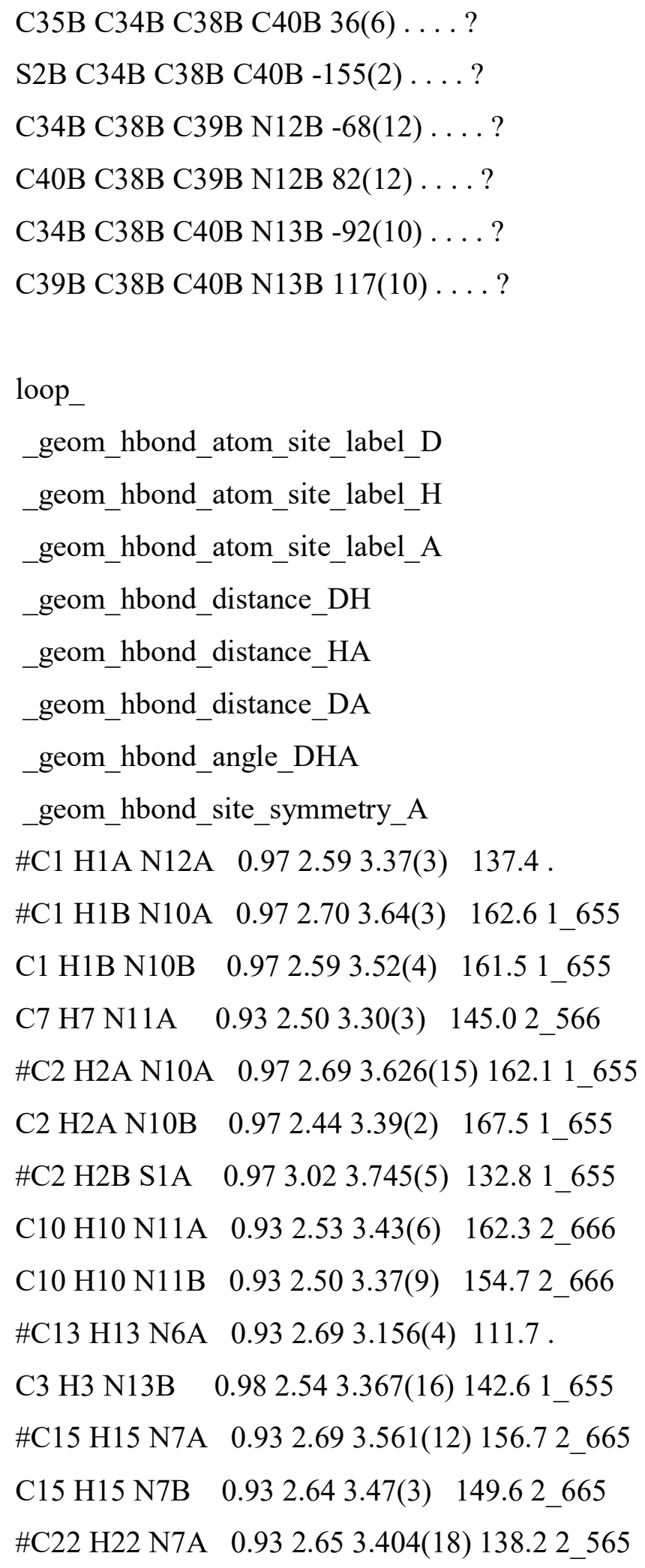


_refine_diff_density_max 0.597

_refine_diff_density_min -0.286

_refine_diff_density_rms 0.045 